\title{
Evaluating the impacts of relaxation and mutation in the SARS-CoV-2 on the COVID-19 epidemic based on a mathematical model: a case study of São Paulo State (Brazil)
}

\author{
Hyun Mo Yang ${ }^{1}$. Luis Pedro Lombardi Junior ${ }^{1}$. Fabio Fernandes Morato Castro ${ }^{2}$. \\ Ariana Campos Yang ${ }^{2}$
}

Received: 4 March 2021 / Revised: 28 September 2021 / Accepted: 29 September 2021 /

Published online: 15 October 2021

(c) SBMAC - Sociedade Brasileira de Matemática Aplicada e Computacional 2021

\begin{abstract}
At the beginning of 2020, SARS-CoV-2 spread to all continents, and many countries adopted quarantine to flatten the COVID-19 outbreak, resulting in the containment of transmission. However, the persistent transmission of SARS-CoV-2, an RNA virus, resulted in the appearance of mutations in the original virus. The appearance of more virulent mutations in different regions of the world resulted in an increased number of severe COVID-19 cases. A mathematical model based on the COVID-19 natural history encompassing the mutations was applied to evaluate the SARS-CoV-2 epidemic. The model parameters were fitted against the observed data from São Paulo State (Brazil) considering quarantine, relaxation, and mutation. The estimated data from São Paulo State showed that the original SARS-CoV-2 transmission better explained the COVID-19 epidemic during the quarantine. However, the increased number of fatalities beyond that predicted by the relaxation indicated the occurrence of mutations. Hence, the relaxation in association with the transmission of a more virulent SARS-CoV-2 better estimated the COVID-19 epidemic from February 26, 2020, to June 15, 2021, in São Paulo State.
\end{abstract}

Communicated by Juan Carlos Cortes.

Hyun Mo Yang

hyunyang@ime.unicamp.br

Luis Pedro Lombardi Junior

luispedro_jr@hotmail.com

Fabio Fernandes Morato Castro

ffmcastro@gmail.com

Ariana Campos Yang

arianacy@gmail.com

1 Department of Applied Mathematics, State University of Campinas, Praça Sérgio Buarque de Holanda, 651, Campinas, SP CEP 13083-859, Brazil

2 Division of Allergy and Immunology, General Hospital of the Medicine School of University of São Paulo, Av. Dr. Eneas Carvalho de Aguiar, 255, São Paulo, SP CEP 05403-000, Brazil 
Keywords Epidemiological scenarios · Mathematical model $\cdot$ SARS-CoV-2 and COVID-19 - Quarantine and relaxation · Mutation and vaccination

Mathematics Subject Classification 92B05 $\cdot 37$ N25

\section{Introduction}

The World Health Organization (WHO) declared coronavirus disease 2019 (COVID-19) a pandemic on March 11, 2020. The severe acute respiratory syndrome coronavirus 2 (SARS$\mathrm{CoV}-2$ ) can be transmitted by droplets that escape lungs through coughing or sneezing and infects humans (direct transmission), or is deposited in surfaces and infects humans when in contact with these contaminated surfaces (indirect transmission) (Liu et al. 2020; Zhu et al. 2020). SARS-CoV-2 infects susceptible individuals through the nose, mouth, or eyes and infects cells in the respiratory tract. COVID-19 in mild form presents fever, dry cough, chills, malaise, muscle pain, and sore throat. In moderate form presents fever, respiratory symptoms, and radiographic characteristics, and in severe form manifests dyspnea, low oxygen saturation, and may evolve to multiple organ failure.

In the absence of effective treatment and vaccine, many countries adopted quarantine to control the SARS-CoV-2 with high transmissibility and lethality at the beginning of the epidemic. Italy (Battineni et al. 2020) and Spain (Guirao 2020) adopted rigid quarantine; however, São Paulo State (Brazil) implemented partial quarantine. In Yang et al. (2021a), a mathematical model based on the COVID-19 natural history encompassing the different fatality rates in young (60 years old or less) and elder (60 years old or more) subpopulations was developed. That model assumed that asymptomatic, pre-symptomatic, and mild COVID19 individuals transmit SARS-CoV-2, while severe COVID-19 cases are isolated in hospitals. It is worth stressing that the SEIR-type (susceptible, exposed, infectious, and recovered compartments) models estimate the transmission rate using the severe COVID-19 registered cases as SARS-CoV-2 transmitters (Yang et al. 2020a). That model was applied to evaluate the impacts on the COVID-19 epidemic by partial quarantine in São Paulo State and rigid quarantine (lockdown) in Spain associated with the protective measures (washing hands with alcohol and gel, use of face mask, and social distancing). Notwithstanding, the relaxation of the isolated population raises the question of better release strategies to avoid the collapse of the health care system. We applied the model presented in Yang et al. (2021a) to forecast the epidemiological scenarios of relaxation (Yang et al. 2020b) based on the plan elaborated by the São Paulo State authorities (Plano são paulo 2020). They assessed the impact of SARS$\mathrm{CoV}-2$ transmission on the healthcare system's capacity. Unfortunately, that relaxation plane was not implemented due to the quick increase in new cases.

Ferguson et al. (2020) adapted an agent-based model describing the influenza epidemic to investigate the impact of the isolation of susceptible individuals on the COVID-19 epidemic, which was referred to as non-pharmaceutical interventions (mitigation and suppression). Briefly, mitigation reduces the basic reproduction number $R_{0}$ but not lower than one, while suppression reduces the basic reproduction number lower than one. Hence, São Paulo State is an example of mitigation, while Spain, of suppression (Yang et al. 2021a). However, the mitigation strategy by a partial quarantine sustains the COVID-19 epidemic. Consequently, like all RNA-based viruses, SARS-CoV-2 suffers mutations as the chain of infections is maintained continuously (WHO 2021). "A virus with one or several new mutations (small changes during the replication) is referred to as a variant of the original virus" (WHO 2021). 
For instance, three variants are challenging the vaccination's control efforts-B.1.1.7 (United Kingdom), B.1.351 (South Africa), and P.1 (Brazil) (see CDC: Sars-cov-2 variants 2021 and references therein). Many countries closed their frontiers, mostly travelers from United Kingdom, South Africa, and Brazil, to control these variants. It is currently accepted that the more virulent variants of SARS-CoV-2 are also more transmissible based on the increased deaths caused by these strains (Korber et al. 2020).

Here, we adapt the model presented in Yang et al. (2021a) to describe the epidemiological scenario of relaxation and SARS-CoV-2 mutations using the COVID-19 cases and deaths registered from São Paulo State (Boletim completo 2020). The adapted model does not take into account the different COVID-19 fatalities depending on age but considers relaxation characterized by a series of pulses' release, SARS-CoV-2 mutation resulting in a more virulent variant, and the waning of immunity. However, this model does not take into account the vaccination. We aim to describe the epidemiological scenario of incipient vaccination found in São Paulo State. Siddiqi and Mehra (2020) described three progressive clinical phases of COVID-19: (1) mild (early infection), (2) moderate, subdivided into without and with hypoxia (pulmonary involvement), and (3) severe (systemic hyper inflammation), leading to the cytokine storm. We assess the number of fatalities taking into account the severe COVID-19 evolution distributed into three clinical phases.

The paper is structured as follows. In Sect. 2, a model is presented to describe the relaxation and SARS-CoV-2 mutation. In Sect. 3, the model is applied to describe the epidemiological scenario using data from São Paulo State. Section 4 presents discussions, and conclusion is given in Sect. 5.

\section{Materials and methods}

The appearance of the mutations occurs as the epidemic evolves. These mutations constitute a pool that can maintain the virulence similar to the original SARS-CoV-2, or more virulent mutations can occur, forming variants with enhanced capacity of infecting cells. A model considering the SARS-CoV-2 mutation resulting in a more virulent variant and the waning of immunity is formulated based on a previous model (Yang et al. 2021a).

\subsection{The SQEAPMDR $1,2,3$ model}

The severe acute respiratory syndrome coronavirus 2 (SARS-CoV-2) infects an individual and initiates its replicates rapidly. As an RNA virus, SARS-CoV-2 can mutate in the process of the cells' infection releasing and originated more virulent variants. We formulate a mathematical model to describe the appearance of more virulent variants from the populational point of view. We assume that all individuals harbor an original type of SARS-CoV-2 initially, and a more virulent one appears later. Hence, we deal with two subpopulations, one harboring original or a pool of less virulent variants and the other, a pool of more virulent variants.

The SQEAPMDR $1,2,3$ model divides the SARS-CoV-2 mutations into pools of a less (original virus, hereafter) and a more (variant virus, hereafter) virulent virus. The model is formulated based on the SARS-CoV-2 transmission flowchart shown in Fig. 1 dividing the population into two subpopulations harboring original and variant (subscript $v$ ) SARS-CoV2.

We describe the subpopulation harboring less virulent according to the natural history of COVID-19. All individuals are divided into susceptible $S$, susceptible individuals who are 


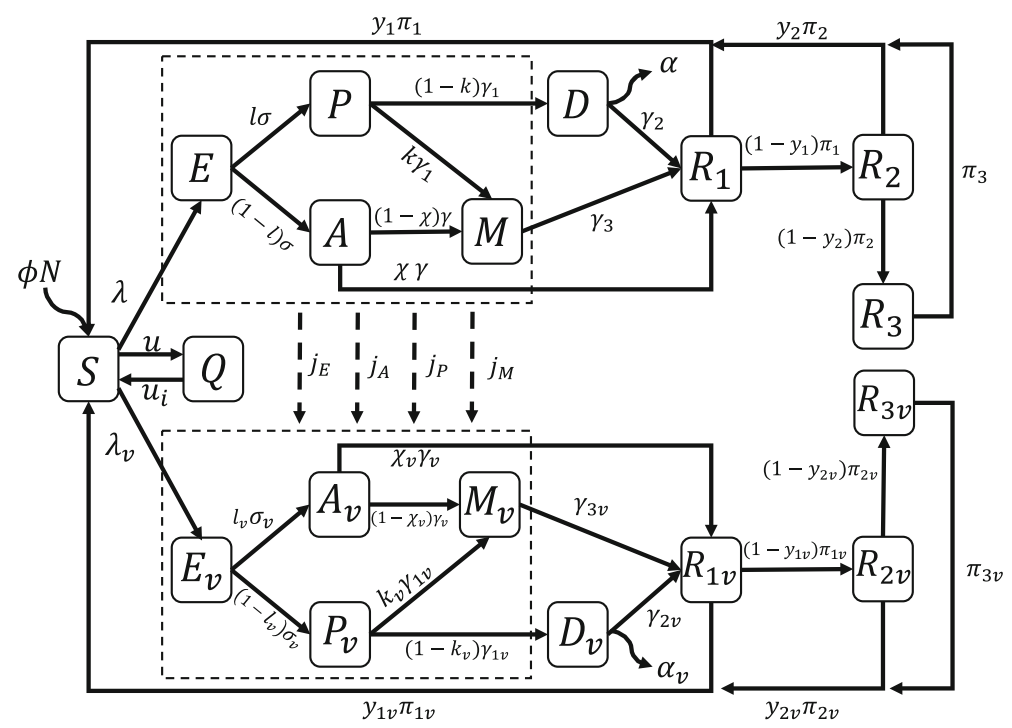

Fig. 1 The flowchart of the SARS-CoV-2 transmission model. The arrow representing natural mortality rate $\mu$ is omitted in all classes

Table 1 Summary of the model variables

\begin{tabular}{ll}
\hline Symbol & Meaning \\
\hline$S$ & Susceptible individuals \\
$Q$ & Isolated individuals \\
$E\left(E_{v}\right)$ & Exposed and incubating SARS-CoV-2 individuals \\
$A\left(A_{v}\right)$ & Asymptomatic individuals \\
$P\left(P_{v}\right)$ & Pre-symptomatic (pre-diseased) individuals \\
$M\left(M_{v}\right)$ & Mild (non-hospitalized) COVID-19 individuals \\
$D\left(D_{v}\right)$ & Severe (hospitalized) COVID-19 individuals \\
$R_{j}\left(R_{j v}\right)$ & Recovered (immune) individuals at stage $j=1,2,3$
\end{tabular}

The subscript $v$ stands for more virulent (variant) subpopulation

isolated $Q$, exposed and incubating $E$, asymptomatic $A$, pre-symptomatic (or pre-diseased, before the onset of COVID-19) $P$, symptomatic with mild $M$ and severe COVID-19 $D$, and recovered (immune) $R_{1}, R_{2}$, and $R_{3}$ compartments. For the more virulent pool, we use the subscript $v$. Table 1 summarizes the model compartments (dynamic variables).

The natural history of SARS-CoV-2 infection is the same for both subpopulations. We assume that individuals in the asymptomatic ( $A$ and $A_{v}$ ), pre-symptomatic $\left(P\right.$ and $P_{v}$ ), and a fraction $z$ (and $z_{v}$ ) of mild COVID-19 $\left(M\right.$ and $M_{v}$ ) compartments are transmitting the virus. Other infected classes $\left((1-z) M, D,\left(1-z_{v}\right) M_{v}\right.$, and $\left.D_{v}\right)$ are under voluntary or forced isolation. Susceptible individuals are infected at a rate $\lambda S$ or $\lambda_{v} S$ (known as the mass action law Anderson and May 1992) and enter into compartment $E$ or $E_{v}$, where $\lambda$ and $\lambda_{v}$ are the per-capita incidence rates (or force of infection) of less and more virulent variants defined by 


$$
\lambda=\frac{\varepsilon}{N}\left(\beta_{1} A+\beta_{2} P+\beta_{3} z M\right), \text { and } \lambda_{v}=\frac{\varepsilon}{N}\left(\beta_{1 v} A_{v}+\beta_{2 v} P_{v}+\beta_{3 v} z_{v} M_{v}\right),
$$

where $\beta_{1}, \beta_{2}$ and $\beta_{3}$ (and $\beta_{1 v}, \beta_{2 v}$ and $\beta_{3 v}$ ) are the transmission rates, that is, the rates at which a virus encounters a susceptible people and infects him/her. The protection factor $\varepsilon \leq 1$ decreases the transmission of infection by individual (face mask, hygiene) and collective (social distancing) protective measures.

Susceptible individuals are infected by original SARS-CoV-2 at a rate $\lambda$ and enter into compartment $E$. After an average period $1 / \sigma$ in the compartment $E_{j}$, where $\sigma$ is the incubation rate, exposed individuals enter into the asymptomatic compartment $A$ (with probability $l$ ) or pre-symptomatic compartment $P$ (with probability $1-l$ ). Possibly asymptomatic individuals can manifest symptoms at the end of the period $1 / \gamma$, where $\gamma$ is the recovery rate of asymptomatic individuals, and a fraction $1-\chi$ enters into the mild COVID-19 compartment $M$. Hence, a fraction $\chi$ enters into the immune (recovered) compartment $R$. For pre-symptomatic individuals, after an average period $1 / \gamma_{1}$ in the compartment $P$, where $\gamma_{1}$ is the infection rate of pre-diseased individuals, these individuals enter into the severe $D$ (with probability $1-k$ ) or mild $M$ (with probability $k$ ) COVID-19 compartments. Severe COVID-19 cases die under the disease-induced (additional) mortality rate $\alpha$. Individuals in compartments $M$ and $D$ acquire immunity after periods $1 / \gamma_{2}$ and $1 / \gamma_{3}$, where $\gamma_{2}$ and $\gamma_{3}$ are the recovery rates of severe and mild COVID-19, and enter into the first immune compartment $R_{1}$. Recovered individuals stay in the compartment $R_{1}$ during an average period $1 / \pi_{1}$, where $\pi_{1}$ is the first waning immunity rate. A fraction $y_{1}$ of these individuals loses immunity returning to the compartment $S$, and $1-y_{1}$ remains immune entering into compartment $R_{2}$. Similarly, from compartment $R_{2}$ flows to $R_{3}$. All individuals in compartment $R_{3}$ wane immunity at a rate $\pi_{3}$.

The more virulent SARS-CoV-2 appears by mutation. Populationally, we assume that a fraction $j_{E}$ among individuals exposed with original type $E$ suffers mutations and enters into the compartment $E_{v}$. Similarly, fractions $j_{A}, j_{P}$, and $j_{M}$ mutate from compartments $A$, $P$, and $M$, entering into compartments $A_{v}, P_{v}$, and $M_{v}$. These infected individuals transmit the variant virus at a rate $\lambda_{v} S$. Once in the compartment $E_{v}$, the flows are similar to those described in the original SARS-CoV-2 natural history. All parameters related to the less virulent variant are applied to the more virulent SARS-CoV-2 differentiated by the subscript $v$.

In the model, we consider pulse isolation and intermittent (series of pulses) release. We assume that there is a unique pulse in isolation at time $t=\tau$, described by $\operatorname{Su} \delta(t-\tau)$, and the intermittent releases are described by $\sum_{i} Q u_{i} \delta\left(t-t_{i}\right)$, where $t_{i}=\tau+\sum_{w=1}^{i} \tau_{w}$. The Dirac delta function $\delta(x)$ is defined by $\delta(x)=\infty$, if $x=0$, otherwise, $\delta(x)=0$, with $\int_{0}^{\infty} \delta(x) \mathrm{d} x=1$. The fraction of individuals in isolation is $u$, and $u_{i}, i=1,2, \cdots$, is the fraction of $i$-th release of isolated individuals, with $\tau_{w}$ being the period between successive releases.

Based on the above descriptions summarized in Fig. 1, the SARS-CoV-2 transmission model is described by the system of ordinary differential equations. Equations for susceptible and isolated individuals are

$$
\left\{\begin{aligned}
\frac{\mathrm{d}}{\mathrm{d} t} S= & \phi N-\mu S-\left(\lambda+\lambda_{v}\right) S+\pi_{1} y_{1} R_{1}+\pi_{1 v} y_{1 v} R_{1 v}+\pi_{2} y_{2} R_{2}+\pi_{2 v} y_{2 v} R_{2 v} \\
& +\pi_{3} R_{3}+\pi_{3 v} R_{3 v}-S u \delta(t-\tau)+\sum_{i} Q u_{i} \delta\left(t-\tau-\sum_{w=1}^{i} \tau_{w}\right) \\
\frac{\mathrm{d}}{\mathrm{d} t} Q= & -\mu Q+S u \delta(t-\tau)-\sum_{i} Q u_{i} \delta\left(t-\tau-\sum_{w=1}^{i} \tau_{w}\right),
\end{aligned}\right.
$$


for original virus harboring individuals,

$$
\left\{\begin{aligned}
\frac{\mathrm{d}}{\mathrm{d} t} E & =\lambda S-(\sigma+\mu) E \\
\frac{\mathrm{d}}{\mathrm{d} t} A & =\left(1-j_{E}\right) l \sigma E-(\gamma+\mu) A \\
\frac{\mathrm{d}}{\mathrm{d} t} P & =\left(1-j_{E}\right)(1-l) \sigma E-\left(\gamma_{1}+\mu\right) P \\
\frac{\mathrm{d}}{\mathrm{d} t} M & =\left(1-j_{A}\right)(1-\chi) \gamma A+\left(1-j_{P}\right) k \gamma_{1} P-\left(\gamma_{3}+\mu\right) M \\
\frac{\mathrm{d}}{\mathrm{d} t} D & =\left(1-j_{P}\right)(1-k) \gamma_{1} P-\left(\gamma_{2}+\mu+\alpha\right) D \\
\frac{\mathrm{d}}{\mathrm{d} t} R_{1} & =\left(1-j_{A}\right) \chi \gamma A+\left(1-j_{M}\right) \gamma_{3} M+\gamma_{2} D-\left(\pi_{1}+\mu\right) R_{1} \\
\frac{\mathrm{d}}{\mathrm{d} t} R_{2} & =\left(1-y_{1}\right) \pi_{1} R_{1}-\left(\pi_{2}+\mu\right) R_{2} \\
\frac{\mathrm{d}}{\mathrm{d} t} R_{3} & =\left(1-y_{2}\right) \pi_{2} R_{2}-\left(\pi_{3}+\mu\right) R_{3},
\end{aligned}\right.
$$

and variant virus harboring individuals,

$$
\left\{\begin{aligned}
& \frac{\mathrm{d}}{\mathrm{d} t} E_{v}=\lambda_{v} S-\left(\sigma_{v}+\mu\right) E_{v} \\
& \frac{\mathrm{d}}{\mathrm{d} t} A_{v}=j_{E} l \sigma E+l_{v} \sigma_{v} E_{v}-\left(\gamma_{v}+\mu\right) A_{v} \\
& \frac{\mathrm{d}}{\mathrm{d} t} P_{v}=j_{E}(1-l) \sigma E+\left(1-l_{v}\right) \sigma_{v} E_{v}-\left(\gamma_{1 v}+\mu\right) P_{v} \\
& \frac{\mathrm{d}}{\mathrm{d} t} M_{v}=j_{A}(1-\chi) \gamma A+j_{P} k \gamma_{1} P+\left(1-\chi_{v}\right) \gamma_{v} A_{v}+k_{v} \gamma_{1 v} P_{v}-\left(\gamma_{3 v}+\mu\right) M_{v} \\
& \frac{\mathrm{d}}{\mathrm{d} t} D_{v}=j_{P}(1-k) \gamma_{1} P+\left(1-k_{v}\right) \gamma_{1 v} P_{v}-\left(\gamma_{2 v}+\mu+\alpha_{v}\right) D_{v} \frac{\mathrm{d}}{\mathrm{d} t} R_{1 v}=j_{A} \chi \gamma A+j_{M} \gamma_{3} M+\chi_{v} \gamma_{v} A_{v}+\gamma_{3 v} M_{v}+\gamma_{2 v} D_{v}-\left(\pi_{1 v}+\mu\right) R_{1 v} \\
& \frac{\mathrm{d}}{\mathrm{d} t} R_{2 v}=\left(1-y_{1 v}\right) \pi_{1 v} R_{1 v}-\left(\pi_{2 v}+\mu\right) R_{2 v} \\
& \frac{\mathrm{d}}{\mathrm{d} t} R_{3 v}=\left(1-y_{2 v}\right) \pi_{2 v} R_{2 v}-\left(\pi_{3 v}+\mu\right) R_{3 v},
\end{aligned}\right.
$$

where $N_{o}=S+Q+E+A+P+M+D+R_{1}+R_{2}+R_{3}$ and $N_{v}=E_{v}+A_{v}+P_{v}+$ $M_{v}+D_{v}+R_{1 v}+R_{2 v}+R_{3 v}$, with $N=N_{o}+N_{v}$ obeying

$$
\frac{\mathrm{d}}{\mathrm{d} t} N=(\phi-\mu) N-\left(\alpha D+\alpha_{v} D_{v}\right) .
$$

This community's vital dynamic is described by the per-capita rates of birth $(\phi)$ and death ( $\mu$ ).

Table 2 summarizes the model parameters and values. The subscript $v$ stands for the variant virus transmitting parameters.

The initial and boundary conditions for the system of Eqs. (2 ), (3), and (4) are given in Appendix A.1. The basic reproduction number $R_{0}$ is presented in Appendix A.2. 
Table 2 Summary of the model parameters and values (rates in days ${ }^{-1}$, time in days and proportions are dimensionless)

\begin{tabular}{|c|c|c|}
\hline Symbol & Meaning & Value \\
\hline$\mu$ & Natural mortality rate & $1 /(78.4 \times 365)^{*}$ \\
\hline$\phi$ & Birth rate & $1 /(78.4 \times 365)^{*}$ \\
\hline$\sigma\left(\sigma_{v}\right)$ & Incubation rate & $1 / 5(1 / 4)^{*}$ \\
\hline$\gamma\left(\gamma_{v}\right)$ & Recovery rate of asymptomatic individuals & $1 / 12(1 / 14)^{*}$ \\
\hline$\gamma_{1}\left(\gamma_{1 v}\right)$ & Infection rate of pre-symptomatic individuals & $1 / 4(1 / 3)^{*}$ \\
\hline$\gamma_{2}\left(\gamma_{2 v}\right)$ & Recovery rate of severe COVID-19 & $1 / 13(1 / 15)^{*}$ \\
\hline$\gamma_{3}\left(\gamma_{3 v}\right)$ & Infection rate of mild COVID-19 individuals & $1 / 13(1 / 15)^{*}$ \\
\hline$\alpha\left(\alpha_{v}\right)$ & Additional mortality rate & $0.0046^{* *}(* *)$ \\
\hline$\pi_{1}\left(\pi_{1 v}\right)$ & Loss of immunity rate at state 1 & $0(0)^{\#}$ \\
\hline$\pi_{2}\left(\pi_{2 v}\right)$ & Loss of immunity rate at state 2 & $0(0)^{\#}$ \\
\hline$\pi_{3}\left(\pi_{3 v}\right)$ & Loss of immunity rate at state 3 & $0(0)^{\#}$ \\
\hline$u$ & Proportion of isolated susceptible individuals & $0.50^{* *}$ \\
\hline$u_{i}$ & Proportion released at time $t_{i}$ & ** \\
\hline$\tau$ & Time of the introduction of isolation & March 24, 2020 \\
\hline$\tau_{i}$ & Times of the $(i$-th) releasing & $* * *$ \\
\hline$\varepsilon$ & Protective factor & $0.52(* *)$ \\
\hline$z\left(z_{v}\right)$ & Proportion of mild COVID-19 transmitter & $0.5(0.6)^{* *}$ \\
\hline$\beta_{1}\left(\beta_{1 v}\right)$ & Transmission rate due to asymptomatic individuals & $0.66(0.66)^{* *}$ \\
\hline$\beta_{2}\left(\beta_{2}\right)$ & Transmission rate due to pre-symptomatic individuals & $0.66(0.66)^{* *}$ \\
\hline$\beta_{3}\left(\beta_{3 v}\right)$ & Transmission rate due to mild COVID-19 individuals & $0.66(0.66)^{* *}$ \\
\hline$j_{E}$ & Proportion of high virulent mutations from class $E$ & *** \\
\hline$j_{A}$ & Proportion of high virulent mutations from class $A$ & $* * *$ \\
\hline$j_{P}$ & Proportion of high virulent mutations from class $P$ & $* * *$ \\
\hline$j_{M}$ & Proportion of high virulent mutations from class $M$ & $* * *$ \\
\hline$y_{1}\left(y_{1 v}\right)$ & Proportion of immunity loss at stage 1 & $0(0)^{\#}$ \\
\hline$y_{2}\left(y_{2 v}\right)$ & Proportion of immunity loss at stage 2 & $0(0)^{\#}$ \\
\hline$\chi\left(\chi_{v}\right)$ & Proportion remaining as asymptomatic individuals & $0.98(0.90)^{*}$ \\
\hline$l\left(l_{v}\right)$ & Proportion of asymptomatic individuals & $0.75\left(0.6^{* *}\right)$ \\
\hline$k\left(k_{v}\right)$ & Proportion of mild (non-hospitalized) COVID-19 & $0.90\left(0.7^{* *}\right)$ \\
\hline
\end{tabular}

Some values are obtained from the literature $\left({ }^{*}\right)$, or estimated $\left({ }^{* *}\right)$, or varied $\left({ }^{* * *}\right)$, or not considered $\left({ }^{\#}\right)$. The subscript $v$ stands for more virulent subpopulation

\subsection{Epidemiological values}

To better describe the epidemiological scenarios of partial quarantine and relaxation, we calculate the following epidemiological values solving the system of Eqs. (2), (3), and (4).

(1) Susceptible individuals - The number of circulating plus isolated susceptible individuals $S^{\text {tot }}$ is obtained from the numbers of non-isolated (circulating) susceptible $(S)$ and isolated $(Q)$ individuals by

$$
S^{\text {tot }}=S+Q
$$


(2) New cases - We calculate the numbers of accumulated severe COVID-19 cases in subpopulations harboring original $\left(\Omega_{o}\right)$ and variant $\left(\Omega_{v}\right)$ virus as

$$
\frac{\mathrm{d}}{\mathrm{d} t} \Omega_{o}=(1-m) \gamma_{1} P \text { and } \frac{\mathrm{d}}{\mathrm{d} t} \Omega_{v}=\left(1-m_{v}\right) \gamma_{1 v} P_{v},
$$

which are given by the exits from $P$ and $P_{v}$, and entering into compartments $D$ and $D_{v}$, with $\Omega=\Omega_{o}+\Omega_{v}$, where $\Omega_{o}(0)=\Omega_{o 0}$ and $\Omega_{v}(0)=\Omega_{v 0}$. The daily severe COVID-19 cases $\Omega_{d}$ is, considering $\Delta t=t_{i}-t_{i-1}=1$ day,

$$
\Omega_{d}\left(t_{i}\right)=\int_{t_{i-1}}^{t_{i}} \frac{\mathrm{d}}{\mathrm{d} t} \Omega \mathrm{d} t=\Omega\left(t_{i}\right)-\Omega\left(t_{i-1}\right),
$$

where $\Omega_{d}(0)=\Omega_{d 0}$ is the first observed COVID-19 case at $t_{0}=0$, with $i=1,2, \cdots$.

(3) Fatalities-The accumulated number of deaths due to severe COVID-19 cases is calculated by $\Theta=\Theta_{o}+\Theta_{v}$, where

$$
\frac{\mathrm{d}}{\mathrm{d} t} \Theta_{o}=\alpha D \text { and } \frac{\mathrm{d}}{\mathrm{d} t} \Theta_{v}=\alpha_{v} D_{v}
$$

which account the exits from compartments $D$ and $D_{v}$ by COVID-19 induced deaths, with $\Theta_{o}(0)=0$ and $\Theta_{v}(0)=0$. In the estimation of the additional mortality rates $\alpha$ and $\alpha_{v}$, we must bear in mind that the time at which new cases and deaths were registered does not have direct correspondence, rather they are delayed by $\Delta$ days, that is, $\Theta_{o}(t+\Delta)=\alpha D(t)$, for instance. The daily COVID-19 deaths $\Theta_{d}$ is, considering $\Delta t=t_{i}-t_{i-1}=1$ day,

$$
\Theta_{d}\left(t_{i}\right)=\int_{t_{i-1}}^{t_{i}} \frac{\mathrm{d}}{\mathrm{d} t} \Theta \mathrm{d} t=\Theta\left(t_{i}\right)-\Theta\left(t_{i-1}\right),
$$

with $\Theta_{d}(0)=0$.

However, instead of calculating the number of deaths based on the severe COVID-19 cases $D$ (dynamic system), we can use the three stages of COVID-19 clinical evolution (Pericàs et al. 2020; Siddiqi and Mehra 2020). From Eq. (7), we can derive the number of individuals in viremia staying short-time in hospital (outpatient) $B_{1}$ (phase 1), the number of patients with inflammatory response needing hospital care (inpatient) $B_{2}$ (phase 2), and the number of patient with cytokine storm needing ICUs (ICUs patient) $B_{3}$ (phase 3). From the numbers $B_{1}, B_{2}$, and $B_{3}$, we obtain the number of deaths due to COVID-19 and the number of cured individuals $C$.

Concerning the parameters of $B_{j}, \Theta_{j}$, and $C_{j}, j=o, v$, a proportion $h_{j}$ of pre-disease individuals enter into phase 1 (outpatients, class $B_{1}$ ), from which a proportion $h_{1 j}$ enters into phase 2 (inpatients, class $B_{2}$ ), and a proportion $h_{2 j}$ enters into phase 3 (ICUs patients, class $B_{3}$ ). The average periods of individuals staying in phases 1,2 , and 3 are $1 / \varsigma_{1 j}, 1 / \varsigma_{2 j}$, and $1 / \varsigma_{3 j}$, where $\varsigma_{1 j}$ and $\varsigma_{2 j}$ are the disease progression rates from phase 1 to 2 and phase 2 to 3 , and $\varsigma_{3 j}$ is the rate of exiting from phase 3 . The additional mortality (fatality) proportions among patients in phases 1,2 , and 3 are $\alpha_{1 j}, \alpha_{2 j}$, and $\alpha_{3 j}$. The fraction $1-h_{j}$ is the nonhospitalization of pre-disease COVID-19 cases $P$, and $1-h_{1 j}-\alpha_{1 j}, 1-h_{2 j}-\alpha_{2 j}$, and $1-\alpha_{3 j}$ are the proportions of cured patients in phases 1,2 , and 3 .

(1) Clinical evolution-The number of outpatients is $B_{1}=B_{1 o}+B_{1 v}$, where

$$
\frac{\mathrm{d}}{\mathrm{d} t} B_{1 o}=h_{o} \frac{\mathrm{d}}{\mathrm{d} t} \Omega_{o}-\left(\mu+\varsigma_{1 o}\right) B_{1 o} \text { and } \frac{\mathrm{d}}{\mathrm{d} t} B_{1 v}=h_{v} \frac{\mathrm{d}}{\mathrm{d} t} \Omega_{v}-\left(\mu+\varsigma_{1 v}\right) B_{1 v},
$$


with $B_{1 o}(0)=B_{1 o 0}$ and $B_{1 v}(0)=0$, and $d \Omega_{j} / \mathrm{d} t, j=o, v$, is given by Eq. (7). The number of inpatients is $B_{2}=B_{2 o}+B_{2 v}$, where

$$
\frac{\mathrm{d}}{\mathrm{d} t} B_{2 o}=h_{1 o} \varsigma_{1 o} B_{1 o}-\left(\mu+\varsigma_{2 o}\right) B_{2 o} \text { and } \frac{\mathrm{d}}{\mathrm{d} t} B_{2 v}=h_{1 v} \varsigma_{1 v} B_{1 v}-\left(\mu+\varsigma_{2 v}\right) B_{2 v}(12)
$$

with $B_{2 o}(0)=0$ and $B_{2 v}(0)=0$. The number of ICUs patients is $B_{3}=B_{3 o}+B_{3 v}$, where

$$
\frac{\mathrm{d}}{\mathrm{d} t} B_{3 o}=h_{2 o} \varsigma_{2 o} B_{2 o}-\left(\mu+\varsigma_{3 o}\right) B_{3 o} \text { and } \frac{\mathrm{d}}{\mathrm{d} t} B_{3 v}=h_{2 v} \varsigma_{2 v} B_{2 v}-\left(\mu+\varsigma_{3 v}\right) B_{3 v}(13
$$

with $B_{3 o}(0)=0$ and $B_{3 v}(0)=0$. The total number of severe COVID-19 cases is $B=B_{1}+B_{2}+B_{3}$.

(2) Fatalities - The number of accumulated deaths caused by severe COVID-19 cases can be calculated from the fatality associated with the clinical evolution divided into $B_{1}, B_{2}$, and $B_{3}$. The number of deaths of outpatients is $\Theta_{1}=\Theta_{1 o}+\Theta_{1 v}$, where

$$
\frac{\mathrm{d}}{\mathrm{d} t} \Theta_{1 o}=\alpha_{1 o} \varsigma_{1 o} B_{1 o} \text { and } \frac{\mathrm{d}}{\mathrm{d} t} \Theta_{1 v}=\alpha_{1 v} \varsigma_{1 v} B_{1 v},
$$

with $\Theta_{1 o}(0)=0$ and $\Theta_{1 v}(0)=0$. The number of deaths of inpatients is $\Theta_{2}=\Theta_{2 o}+\Theta_{2 v}$, where

$$
\frac{\mathrm{d}}{\mathrm{d} t} \Theta_{2 o}=\alpha_{2 o} \varsigma_{2 o} B_{2 o} \text { and } \frac{\mathrm{d}}{\mathrm{d} t} \Theta_{2 v}=\alpha_{2 v} \varsigma_{2 v} B_{2 v},
$$

with $\Theta_{2 o}(0)=0$ and $\Theta_{2 v}(0)=0$. The number of deaths of ICUs patients is $\Theta_{3}=$ $\Theta_{3 o}+\Theta_{3 v}$, where

$$
\frac{\mathrm{d}}{\mathrm{d} t} \Theta_{3 o}=\alpha_{3 o} \varsigma_{3 o} B_{3 o} \text { and } \frac{\mathrm{d}}{\mathrm{d} t} \Theta_{3 v}=\alpha_{3 v} \varsigma_{3 v} B_{3 v},
$$

with $\Theta_{3 o}(0)=0$ and $\Theta_{3 v}(0)=0$. The total number of deaths is $\Theta=\Theta_{1}+\Theta_{2}+\Theta_{3}$. The daily COVID-19 deaths $\Theta_{d}$ can be obtained using Eq. (10).

(3) Cures-The total number of individuals being cured is $C=C_{o}+C_{v}$, where

$$
\left\{\begin{array}{l}
\frac{\mathrm{d}}{\mathrm{d} t} C_{o}=\left(1-h_{1 o}-\alpha_{1 o}\right) \varsigma_{1 o} B_{1 o}+\left(1-h_{2 o}-\alpha_{2 o}\right) \varsigma_{2 o} B_{2 o}+\left(1-\alpha_{3 o}\right) \varsigma_{3 o} B_{3 o}-\mu C_{o} \\
\frac{\mathrm{d}}{\mathrm{d} t} C_{v}=\left(1-h_{1 v}-\alpha_{1 v}\right) \varsigma_{1 v} B_{1 v}+\left(1-h_{2 v}-\alpha_{2 v}\right) \varsigma_{2 v} B_{2 v}+\left(1-\alpha_{3 v}\right) \varsigma_{3 v} B_{3 v}-\mu C_{v},
\end{array}\right.
$$

with $C_{o}(0)=C_{v}(0)=0$.

Table 3 summarizes the parameters related to the clinical evolution of the original virus and values (for the variant virus, the values are given between parentheses). See Appendix B for the assessment of the parameters' values.

\section{Results}

At the beginning of the COVID-19 epidemic, we have the transmission of the original SARS$\mathrm{CoV}-2$, and we assume that only the less virulent viruses are circulating while the quarantine lasts, and the more virulent viruses' transmission initiates with relaxation. In other words, the accumulated mutations occurring during the quarantine manifests populationally in its enhanced virulence when relaxation begins.

We follow the procedure presented in Yang et al. (2021a) to estimate the model parameters using data from February 26, 2020 to June 15, 2021 (Boletim completo 2020) to describe the COVID-19 epidemic in São Paulo State. The model parameters are estimated using Eq. (34) in Appendix B. 
Table 3 Summary of the parameters related to hospitalization and values (rates in days ${ }^{-1}$ and proportions are dimensionless)

\begin{tabular}{|c|c|c|}
\hline Symbol & Meaning & Value \\
\hline$\alpha_{1 o}\left(\alpha_{1 v}\right)$ & Fatality proportion of inpatients in phase 1 & $0.0005\left(^{* *}\right)$ \\
\hline$\alpha_{2 o}\left(\alpha_{2 v}\right)$ & Fatality proportion of inpatients in phase 2 & $0.04(* *)$ \\
\hline$\alpha_{3 o}\left(\alpha_{3 v}\right)$ & Fatality proportion of inpatients in phase 3 & $0.55\left(^{* *}\right)$ \\
\hline$\varsigma_{1 o}\left(\varsigma_{1 v}\right)$ & Progression rate from phase 1 to 2 & $1 / 3(1 / 4)^{*}$ \\
\hline$\varsigma_{2 o}\left(\varsigma_{2 v}\right)$ & Progression rate from phase 2 to 3 & $1 / 7(1 / 9)^{*}$ \\
\hline$\varsigma 3 o(\varsigma 3 v)$ & Rate of exit from phase 3 & $1 / 15(1 / 20)^{*}$ \\
\hline$h_{o}\left(h_{v}\right)$ & Proportion of intense viremia & $1.0(1.0)^{*}$ \\
\hline$h_{1 o}\left(h_{1 v}\right)$ & Proportion of progression from phase 1 to 2 & $0.21^{*}(* *)$ \\
\hline$h_{2 o}\left(h_{2 v}\right)$ & Proportion of progression from phase 2 to 3 & $0.45^{*}(* *)$ \\
\hline
\end{tabular}

Some values are calculated $(*)$ or estimated $\left({ }^{* *}\right)$. The subscript $v$ stands for more virulent subpopulation

\subsection{The epidemiological scenario of quarantine}

The observed data, from February 26, 2020, to June 15, 2021 (Boletim completo 2020), are partitioned into two sets - the first set is used to estimate the model parameters (input data set), and the estimated model is then confronted with the second set (test data set) to assess its prediction ability (Alvarenga et al. 1999). Hence, we estimate the model parameters using severe COVID-19 cases $\left(\Omega^{o b}\right)$ and deaths $\left(\Theta^{o b}\right)$ recorded from February 26 to May 7 , 2020 (input data set). Then, we use the estimated parameters to predict the epidemic under interventions (quarantine and relaxation) from May 8, 2020, to June 15, 2021 (test data set) and compare the model predictions with the observed data.

Using the input data set of registered COVID-19 cases and deaths from São Paulo State (Boletim completo 2020), we estimate the model parameters considering only the original SARS-CoV-2 infection-the partial quarantine (isolation) followed by the protective measures adopted by the population. We split the data set into four periods to estimate the model parameters following those procedures used in Yang et al. (2021a).

(1) Transmission rates (from February 26 to April 3, 2020) -Estimated values are $\beta=$ $\beta_{1}=\beta_{2}=\beta_{3}=0.66$ days $^{-1}$, resulting in the basic reproduction number $R_{0}=7.63$. (Letting $\beta_{v}=\beta_{1 v}=\beta_{2 v}=\beta_{3 v}=0.66$ days $^{-1}$, we should have $R_{0 v}=8.35$ ). We used the initial conditions and $R_{0}$ given by Eqs. (18) and (31) in Appendix A.

(2) Proportion in isolation (from March 24 to April 12, 2020) —Estimated value is $k=0.50$. The onset of severe COVID-19 symptoms appear 9 days after the isolation's introduction (see Yang et al. 2021a). We used the boundary conditions given by Eq. (20) in Appendix A.1.

(3) Protective measures (from April 4 to May 7, 2020)-Estimated value is $\varepsilon=0.52$. The transmission rate $\beta=0.66$ days $^{-1}$ is reduced to $\beta^{\prime}=\varepsilon \beta=0.34$ days $^{-1}$, reducing the basic reproduction number $R_{0}$ to $R_{\text {red }}=3.97$.

(4) Additional mortality rate (from March 16 to May 7, 2020)—Fixing $\Delta=15$ days, the estimated value is $\alpha=0.0046$ days $^{-1}$. The death occurs 15 days after the onset of severe COVID-19 symptoms (see Yang et al. 2021a).

The period from February 26 to May 7, 2020, characterizes the beginning of the epidemic under partial quarantine and protective measures adopted by individuals. Considering a pool of less virulent (original) SARS-CoV-2, we estimated parameters considering the input data 

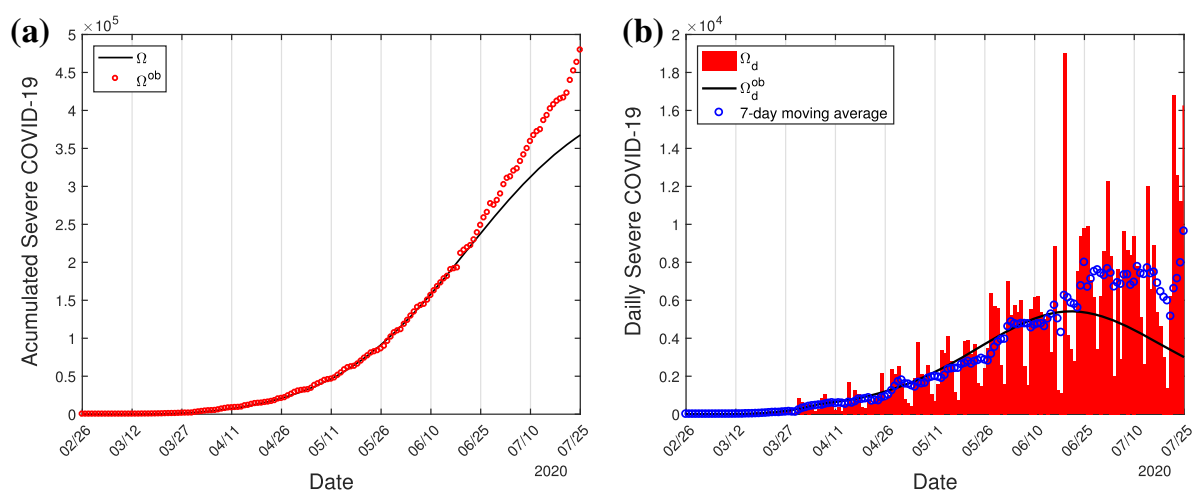

Fig. 2 The curve of estimated $\Omega$ and observed data (a), and the calculated $\Omega_{d}$, the observed data, and the 7-day moving average (b)
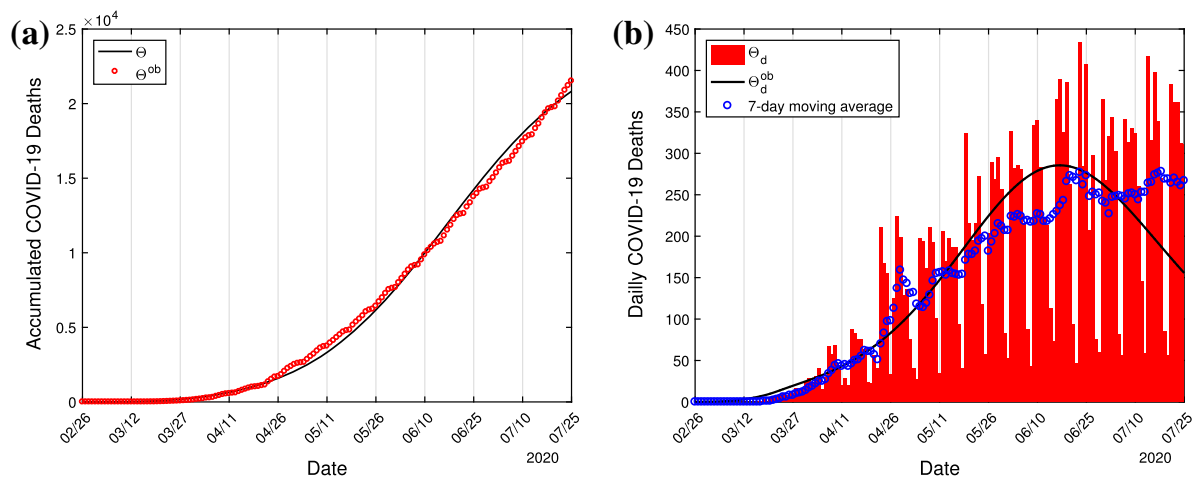

Fig. 3 The estimated curve $\Theta$ and the observed data (a), and the calculated curve $\Theta_{d}$, the observed data, and the 7-day moving average (b)

set. The estimated parameters given in Table 2 are summarized in the following epidemiological scenarios. We compare these curves with the test data set aiming: (1) to study the epidemic's prediction ability, and (2) to include other factors (relaxation and mutation) when data and estimated curves diverge.

Figure 2 shows the estimated curve $\Omega$ and the observed data (a), and the calculated curve $\Omega_{d}$ and the observed data (b). The 7-day moving average of the daily observed data is also shown in Fig. 3b. The curves $\Omega$ and $\Omega_{d}$ are given by Eqs. (7) and (8).

The model parameters were estimated against the severe COVID-19 observed data $\Omega^{o b}$. In Fig. 2a, the first observed point detached from the estimated curve $\Omega$ on June 23, 2020, and all subsequent data situate above the curve. Hence, the original virus parameters' values fit the test data set from May 8 to June 22, 2020.

Using the set of estimated parameters, we simulate the system of Eqs. (2), (3), and (4) to yield the severe COVID-19 epidemic curve $D$. From this curve, using Eq. (9), we estimate the fatality rate $\alpha$ to obtain the accumulated number of deaths $\Theta$. From this estimated curve, we calculate the daily curve $\Theta_{d}$ using Eq. (10). Figure 3 shows the estimated curve $\Theta$ (a) and the calculated curve $\Theta_{d}$ (b) with the corresponding observed data. The 7-day moving average of the daily observed data is also shown in Fig. $3 b$. 

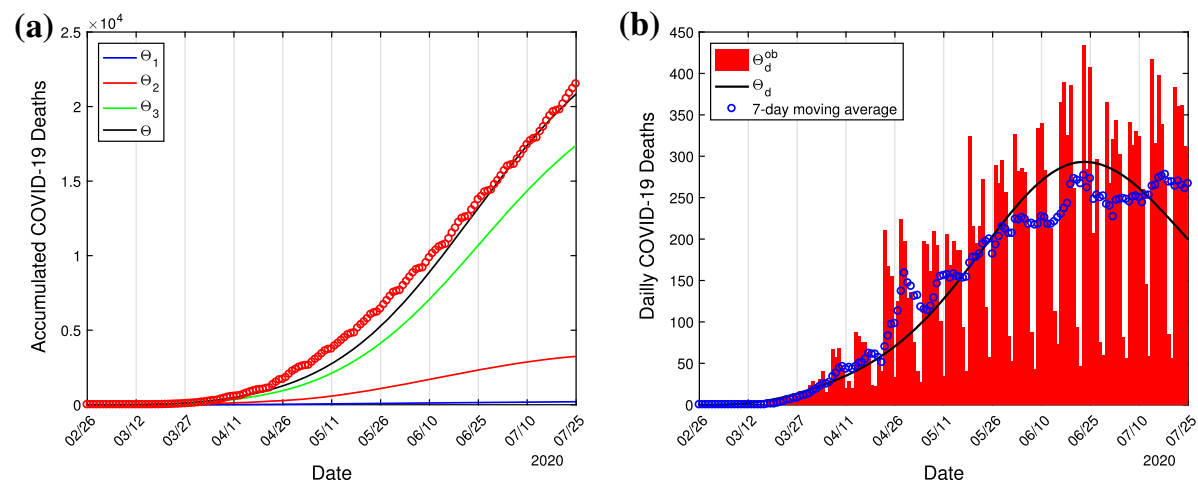

Fig. 4 The estimated curves $\Theta_{1}, \Theta_{2}, \Theta_{3}$, and $\Theta=\Theta_{1}+\Theta_{2}+\Theta_{3}$ (a) and the calculated daily curve of $\Theta_{d}=\Theta\left(t_{i}\right)-\Theta\left(t_{i-1}\right)$, the observed data, and the 7-day moving average (b)

In Fig. 3a, the first observed point detached from the estimated curve $\Theta$ on July 21, 2020, and all subsequent data situate above the curve. Hence, the original virus parameters' values fit the test data set from May 8 to July 20, 2020. Notice that the first observed point detaching from the estimated curve $\Theta$ occurred on July 21, while the severe COVID-19 curve $\Omega$ separated on June 23, 28 days earlier. In the estimation of mortality rate $\alpha$, we used $\Delta=15$ days in $\Theta(t+\Delta)=\alpha D(t)$ and, consequently, it was expected that the curve of $\Omega$ should be detached from the observed data on July 6. Somehow, the unexpectedly early separation demonstrates that the observed COVID-19 cases also bring mild cases, and we cannot assure that the observed data constitute only the severe COVID-19 cases anymore since after. Therefore, the fatality data are more reliable in characterizing the severe COVID19 cases than the observed COVID-19 data containing mild and possibly asymptomatic cases. In other words, we cannot match the registered data with the severe COVID-19 curve $D$.

Let us assess the model's prediction ability by comparing the observed data with the original SARS-CoV-2 epidemiological quarantine scenario. Since July 21, 2020, the observed COVID-19 fatalities situate systematically above the estimated curve $\Theta$, showing that the epidemiological scenario of quarantine was perturbed. (From Figs. 2a, 3a, the epidemiological scenario under quarantine using input data set adjusted the test data set from May 8 to June $22(\Omega)$ or July $20(\Theta)$, showing that quarantine lasted until that date.) Indeed, São Paulo State initiated the relaxation by the end-June 2020. Hence, since July 6, 2020, the test data set must be described by incorporating intermittent pulses of release in the model (Yang and Greenhalgh 2015).

Besides evaluating the deaths from non-specified severe COVID-19 cases $D$, we use the clinical evolution since the onset of symptoms to assess the number of fatalities. The number of deaths is estimated taking into account the three clinical phases of COVID-19. Figure 4 shows the estimated curves $\Theta_{1}, \Theta_{2}, \Theta_{3}$, and $\Theta=\Theta_{1}+\Theta_{2}+\Theta_{3}$ (a) and the calculated daily curve of $\Theta_{d}=\Theta\left(t_{i}\right)-\Theta\left(t_{i-1}\right)$ and the observed data (b). The curve corresponding to $\Theta_{1}$ is close to the horizontal axis. We used the values given in Table 3, and Eqs. (14), (15) and (16) for $\Theta_{1}, \Theta_{2}$, and $\Theta_{3}$. In the estimation of the fatality rate $\alpha_{i o}, i=1,2,3$, we used $\Delta=18$ days.

The epidemiological scenario of partial quarantine in São Paulo State was described by the model parameters' values given in Table 2 and the values for the COVID-19 in hospitals' care given in Table 3. Figures 2 and 3 describe the quarantine considering only the original SARSCoV-2 transmission. In Yang et al. (2020b), the epidemiological scenarios of relaxation's 
Table 4 The $i$-th date and proportion of release $\left(u_{i}\right)$, the protective measure $(\varepsilon)$, the proportion transferring from original to variant viruses $j_{k}^{i}$, with $k=E, A, P, M$, and the fatality rate $\alpha_{v}$

\begin{tabular}{llllllll}
\hline Date & $u_{i}$ & $\varepsilon$ & $j_{E}$ & $j_{A}$ & $j_{P}$ & $j_{M}$ & $\alpha_{v}$ \\
\hline During quarantine & 0 & 0.52 & - & - & - & - & - \\
July 5, 2020 & 0.1 & 0.52 & 0.01 & 0.01 & 0.02 & 0.03 & 0.0052 \\
July 20, 2020 & 0.1 & 0.55 & 0.01 & 0.01 & 0.02 & 0.03 & 0.0052 \\
August 4, 2020 & 0.1 & 0.56 & 0.01 & 0.01 & 0.02 & 0.03 & 0.0052 \\
August 19, 2020 & 0.1 & 0.6 & 0.01 & 0.01 & 0.02 & 0.03 & 0.0052 \\
November 2, 2020 & 0.48 & 0.65 & 0.05 & 0.2 & 0.4 & 0.6 & 0.0052 \\
February 25, 2021 & 0.6 & 0.75 & 0.15 & 0.3 & 0.6 & 0.9 & 0.0064 \\
\hline
\end{tabular}

description were assessed based on the plan elaborated by the S ão Paulo State authorities (Plano são paulo 2020). There, the COVID-19 deaths were under-estimated (see Fig. 16 in Yang et al. (2020b) for severe COVID-19 cases), showing that more virulent viruses must be considered besides the relaxation. The epidemiological scenario in Amazonas State (Brazil) can shed more light on the virulent variants' role in association with relaxation. In Amazonas State, deaths in January-February 2021 are higher than those that occurred during all 2020 (Informações covid-19 2021). This increased number cannot be explained only by the partial relaxation once a significant number of deaths caused by the P.1 variant was found.

\subsection{The epidemiological scenario of relaxation}

In the preceding section, we estimated the model parameters against the severe COVID19 observed data. In the first months after the outbreak of the COVID-19 epidemic, the scarcity of the tests (serology and PCR) due to the novelty of the pandemic did not allow to test those individuals with COVID-19 symptoms. Hence, the test to confirm infection by SARS-CoV-2 was performed basically in the hospitalized and dead individuals presenting COVID-19 symptoms. Thus, almost all recorded data in São Paulo State were composed of severe COVID-19 cases. However, the widespread test to catch SARS-CoV-2 infection included those presenting mild COVID-19 and asymptomatic individuals. For this reason, in this section, we estimate the registered COVID-19 deaths instead of the severe cases.

The parameters related to the natural history of the original COVID-19 and the estimated transmission parameters are given in Table 2, which are maintained constant. The parameters' values for the variant COVID-19 are changed based on the original virus's values, given in Table 2. As we pointed out, the mutations occur as the epidemic evolves, and individuals infected with a pool of less virulent viruses may harbor more virulent viruses by mutation. The variant virus may coexist or even displaces the original virus by competition, and the severe COVID-19 cases increase. Hence, we estimate the fraction of individuals infected with the original virus being transferred to the compartment of individuals harboring variant viruses by mutations. Table 4 shows the date of the $i$-th transfer and the corresponding value for $j_{k}^{i}$, with $k=E, A, P, M$, and the fatality rate $\alpha_{v}$. The fatality rate increased by $14 \%$ compared to the mortality induced by the original virus, which increased by $39 \%$ only in the last release on February 25, 2021.

In Table 4, we assumed small $j_{E}$, and the relations $j_{P}=2 j_{A}$ and $j_{M}=3 j_{A}$. The reason behind these assumptions is the increased mutations occurring along with the progression 

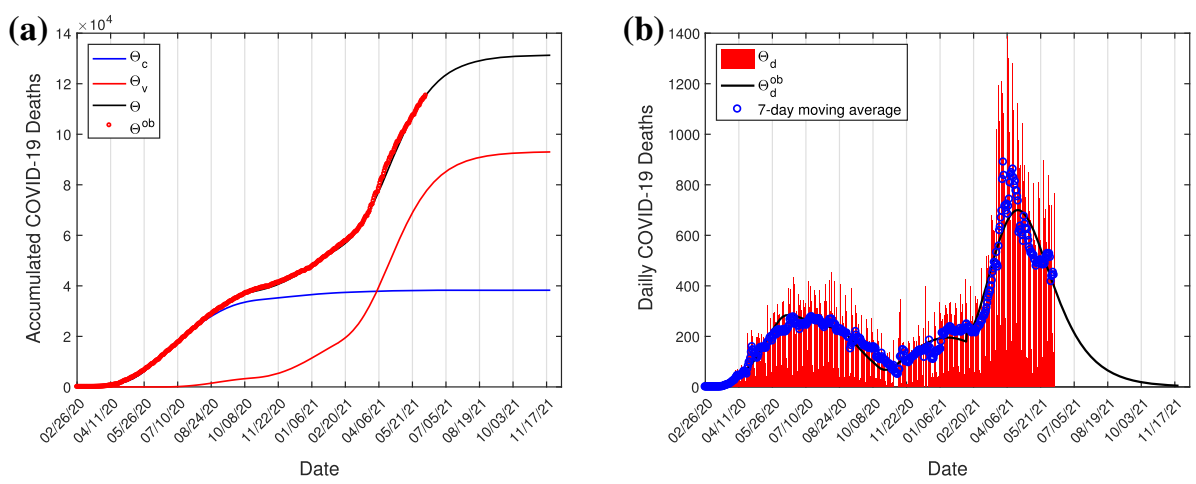

Fig. 5 The estimated curve $\Theta$ and the observed data (a), and the calculated curve $\Theta_{d}$, the observed data, and the 7-day moving average (b)
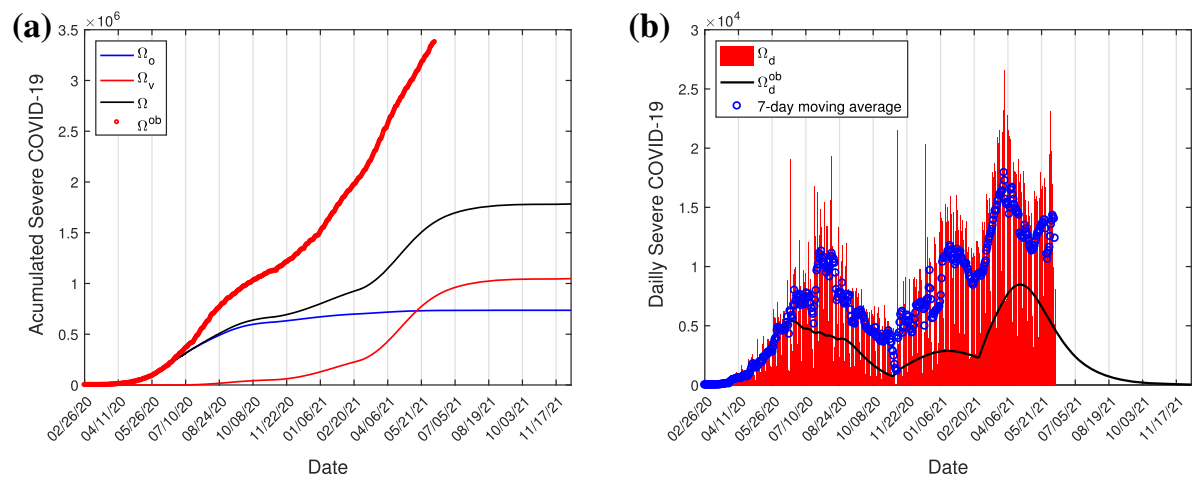

Fig. 6 The curve of estimated $\Omega$ and observed data (a), and the calculated $\Omega_{d}$, observed data, and the 7-day moving average $(b)$

of the infection. For instance, the exposed individuals are just infected and harboring a negligible amount of virus; hence, $j_{E}$ assumes small value. Suppose that a small amount of virus characterizes asymptomatic status; thus, $j_{A}$ must be lower than $j_{P}$ and $j_{M}$. Finally, individuals in mild disease conditions spend more time in this stage than pre-symptomatic individuals besides harboring a large amount of virus; hence $j_{M}>j_{P}$. Notice that the transfer from compartments of individuals infected with the original virus to variant virus compartments increased on November 2, 2020, with an additional increase on February 25, 2021.

Figure 5 shows the estimated curves $\Theta_{o}, \Theta_{v}$, and $\Theta$ and the observed data (a), and the calculated curve $\Theta_{d}$ and the observed data (b). The 7-day moving average of the daily observed data is also shown in Fig. 5b. The curves $\Theta$ and $\Theta_{d}$ are given by Eqs. (9) and (10). The estimated curves $\Theta_{o}, \Theta_{v}$, and $\Theta$ approach asymptotically 38270, 93000, and 131300 .

Figure 6 shows the estimated curves $\Omega_{o}, \Omega_{v}$, and $\Omega$ and the observed data (a), and the calculated curve $\Omega_{d}$ and the observed data (b). The 7-day moving average of the daily observed data is also shown in Fig. 6b. The curves $\Omega$ and $\Omega_{d}$ are given by Eqs. (7) and (8). The estimated curves $\Omega_{o}, \Omega_{v}$, and $\Omega$ approach asymptotically 735800, 1.408 million, and 1.783 million. 

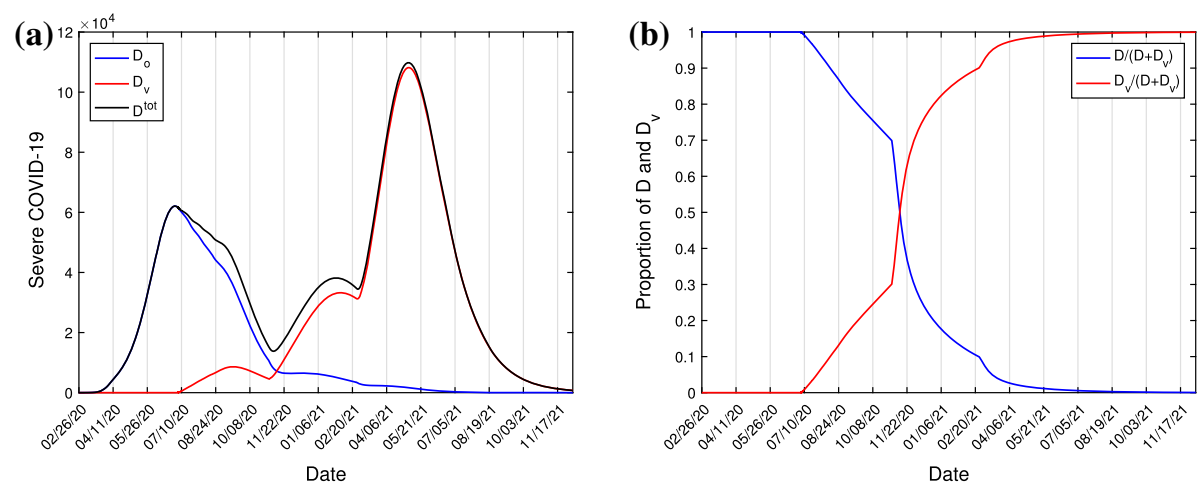

Fig. 7 The estimated curves of $D, D_{v}$, and $D^{\text {tot }}=D+D_{v}(\mathbf{a})$ and the proportions $D / D^{\text {tot }}$ and $D_{v} / D^{\text {tot }}(\mathbf{b})$

Figure 6a shows the recorded COVID-19 data above the estimated severe cases $\Omega$, confirming that these observed data carry on the mild and perhaps asymptomatic individuals caught by mass tests. However, Fig. $6 \mathrm{~b}$ shows roughly a similar trend between the lowervalued estimated daily curve $\Omega_{d}$ and the observed data $\Omega^{o b}$. Notice that at mid-May 2021, there is an increasing trend not captured by the estimation, which was also seen smoothly in Fig. 5b.

Figure 7a shows the COVID-19 epidemic curves $D, D_{v}$, and $D^{\text {tot }}=D+D_{v}$ corresponding to the estimated curves presented in Figs. 5a and 6a. Figure $7 \mathrm{~b}$ shows the proportions $D / D^{\text {tot }}$ and $D_{v} / D^{\text {tot }}(\mathrm{b})$. The ascending phase of the $D$ curve is roughly characterized by the effective reproduction number given by Eq. (33) greater than one $\left(R_{e f}>1\right)$, while the descending phase, by $R_{e f}<1$ (see Yang et al. 2021a). The epidemic peak (62080) with the original virus in the quarantine occurred on July 1, 2020, and the epidemic peak (109800) with the majority of variant virus in the relaxation occurred on May 5, 2021. The more virulent SARS-CoV-2 transmission increased the severe COVID-19 cases by $77 \%$.

Figure 7 a shows a severe variant epidemic increasing rapidly at the end of 2020, reaching the peak on May 5, 2021. This more intense epidemic in comparison with the original one is due to the relaxation allied with the widespread of the variant SARS-CoV-2 having higher $R_{0 v}\left(9.4 \%\right.$ higher than $\left.R_{0}\right)$. In Fig. $7 \mathrm{~b}$, we observe the displacement of the original SARS$\mathrm{CoV}-2$ by the variant virus, which corresponds to around $80 \%$ of infections at the beginning of 2021.

When susceptible individuals are infected, they pass through the natural history of COVID19 stages and become immunes. Figure 8 shows the curves $S, Q$, and $S^{\text {tot }}=S+Q$ (a) and the curves $R, R_{v}$, and $R^{\text {tot }}=R+R_{v}$ (b). The susceptible individuals $S^{\text {tot }}$ decrease from 44.6 million to 6.8 million, while the recovered individuals increase from 0 to 36.7 million. At the end of the epidemic, the susceptible and recovered individuals are $15.25 \%$ and $82.3 \%$ of the population.

We estimated $\Theta$, from which we retrieved $\Omega$. Now, using this $\Omega$, we estimate the COVID19 fatalities curves $\Theta_{1}, \Theta_{2}$, and $\Theta_{3}$ considering the clinical evolution of the disease. Table 5 shows the estimated parameters related to the clinical evolution of the variant virus. In the estimation of the fatality rates $\alpha_{i o}, i=1,2,3$, we used $\Delta=15$ days.

Notice that during the quarantine, we used $\Delta=18$ days to estimate the curves $\Theta_{1}, \Theta_{2}$, and $\Theta_{3}$. However, the appearance of variant SARS-CoV-2 after the relaxation reduced the appearance of the COVID-19 symptoms to $\Delta=15$ days. The fatality rates $\alpha_{i o}, i=1,2,3$, were not changed in comparison with the deaths induced by the original virus but increased 

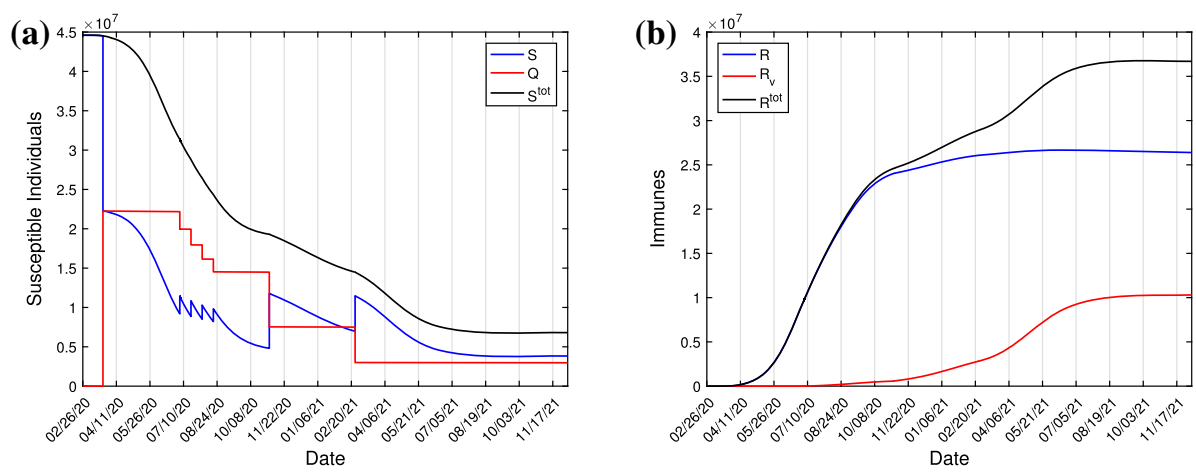

Fig. 8 The estimated curves of $S, Q$, and $S^{\text {tot }}=S+Q(\mathbf{a})$ and the estimated curves of $R, R_{v}$, and $R^{\text {tot }}=R+R_{v}$ (b)

Table 5 The estimated parameters related to the clinical evolution of the variant virus and $\Delta$

\begin{tabular}{lllllll}
\hline Date & $h_{1 v}$ & $h_{2 v}$ & $\alpha_{1 v}$ & $\alpha_{2 v}$ & $\alpha_{3 v}$ & $\Delta$ (days) \\
\hline During quarantine & - & - & - & - & - & 18 \\
July 5, 2020 & 0.25 & 0.5 & 0.0005 & 0.04 & 0.55 & 15 \\
November 2, 2020 & 0.27 & 0.52 & 0.0005 & 0.04 & 0.55 & 15 \\
February 25, 2021 & 0.27 & 0.52 & 0.0006 & 0.055 & 0.57 & 15
\end{tabular}
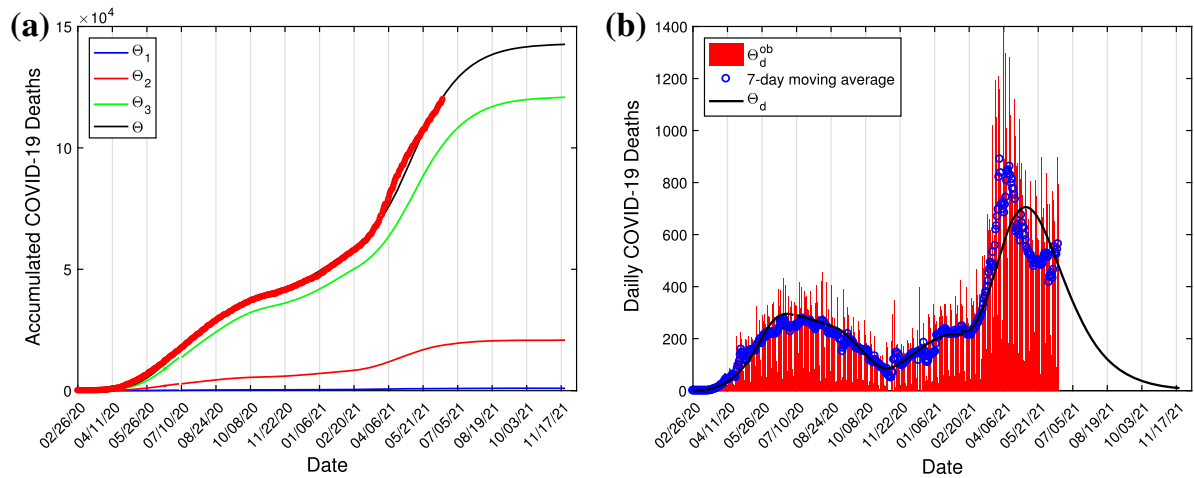

Fig. 9 The estimated curves $\Theta_{1}, \Theta_{2}, \Theta_{3}$, and $\Theta=\Theta_{1}+\Theta_{2}+\Theta_{3}$ (a) and the calculated daily curve of $\Theta_{d}=\Theta\left(t_{i}\right)-\Theta\left(t_{i-1}\right)$, the observed data, and the 7-day moving average (b)

only in the last release on February 25, 2021. However, the proportions $h_{1 v}$ and $h_{2 v}$ increased since the beginning of the relaxation on July 6, 2020.

Figure 9 shows the estimated curves $\Theta_{1}, \Theta_{2}, \Theta_{3}$, and $\Theta=\Theta_{1}+\Theta_{2}+\Theta_{3}$ (a) and the calculated daily curve $\Theta_{d}=\Theta\left(t_{i}\right)-\Theta\left(t_{i-1}\right)$ and the observed data (b). The 7-day moving average of the daily observed data is also shown in Fig. 9b. We used the values given in Table 5, and Eqs. (14), (15) and (16) for $\Theta_{1}, \Theta_{2}$, and $\Theta_{3}$. For daily curve $\Theta_{d}$, we used Eq. (10). The estimated curves $\Theta_{1}, \Theta_{2}, \Theta_{3}$, and $\Theta$ approach asymptotically $973,20830,120800$, and 142600 .

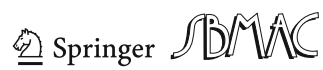


The clinical evolution, using Eqs. (14), (15) and ( 16), estimated 11300 (8.6\%) more fatalities than that provided by the dynamic system, using Eq. (9). Figure $5 \mathrm{~b}$ shows the estimated curve $\Theta$ following the trend of the 7-day moving average of the observed data $\Theta^{o b}$ except in March and April 2021. In March, the data are situated above the curve, but in April, below the data. However, Fig. $9 \mathrm{~b}$ shows this behavior from March to May.

\section{Discussion}

In the first months of the COVID-19 outbreak, the absence of a mass test against SARS-CoV2 infection and clinical follow-up of infected individuals did not permit the discrimination between asymptomatic and pre-symptomatic individuals. Considering this initial period, we explained the COVID-19 data from São Paulo State considering less virulent (original) SARS-CoV-2 transmission. The estimated model parameters yielded $R_{0}=7.63$ for the basic reproduction number corresponding to the original virus. A more complex model incorporating different fatalities among individuals aged up to 60 years and above 60 years resulted in $R_{0}=R_{0 y}+R_{0 o}=9.24$ (partials $R_{0 y}=7.73$ for subpopulation below 60 years, and for above 60 years old, $R_{0 o}=1.51$ ) (Yang et al. 2021a).

The partial quarantine and protective measures flattened the epidemic curve in São Paulo State. Still, the transmission was maintained at a relatively higher level (the effective reproduction number reached one around 80 days later Yang et al. 2021a). However, since July 21,2020 , the observed number of deaths was systematically above the estimated curve $\Theta$ considering quarantine only. This fact permitted establishing that the effects of relaxation began in São Paulo State appeared on July 21; however, it was not fully implemented.

However, the persistent transmission of SARS-CoV-2 should result in a high number of mutations. Among these mutations, some acquired an enhanced ability to infect cells, increasing the risk of death. In fact, São Paulo State still maintained part of the population in isolation, but 68,904 deaths on March 24, 2021, surpassed 57,300 predicted by the epidemic with original SARS-CoV-2 (Yang et al. 2021a). This higher number of deaths may be a consequence of the appearance of more virulent variants during the prolonged SARS-CoV-2 transmission. Moreover, more viruses will be released in the environment when more cells are infected, increasing the transmission. Considering these biological effects, we shortened parameters $\sigma_{v}^{-1}$ and $\gamma_{1 v}^{-1}$ (times to become infectious and to present symptoms) and enlarged $\gamma_{v}^{-1}, \gamma_{2 v}^{-1}$, and $\gamma_{3 v}^{-1}$ (times to recover from the infection) in Table 2. On the other hand, the increased number of severe symptomatic individuals is isolated in hospitals, and mild cases are detected by test and advised to isolate. Contrarily, the decreased numbers of asymptomatic and pre-symptomatic individuals (carriers) are not detected and circulate freely. Additionally, the carriers are in close contact with other susceptible individuals, contrarily to individuals manifesting any suspicious symptoms (not necessarily COVID-19). These populational features and social behaviors may vary the transmission rates, increasing or decreasing. These complex biological and populational influences on the COVID-19 epidemic were oversimplified in our model, and letting for the variant virus transmission rates the same values estimated from the original virus, we obtained $R_{0 v}=8.35$.

In the model, the force of infections $\lambda$ and $\lambda_{v}$ defined by Eq. (1) provide the per-capita incidences in the subpopulations infected with original and variant SARS-CoV-2. We assumed a transfer $\left(j_{E}, j_{A}, j_{P}\right.$, and $\left.j_{M}\right)$ from individuals infected with the original virus to the status of infection with the variant virus. This transfer can be hypothesized to have two origins in the individuals infected with the pool of less virulent virus: (1) the occurrence of muta- 


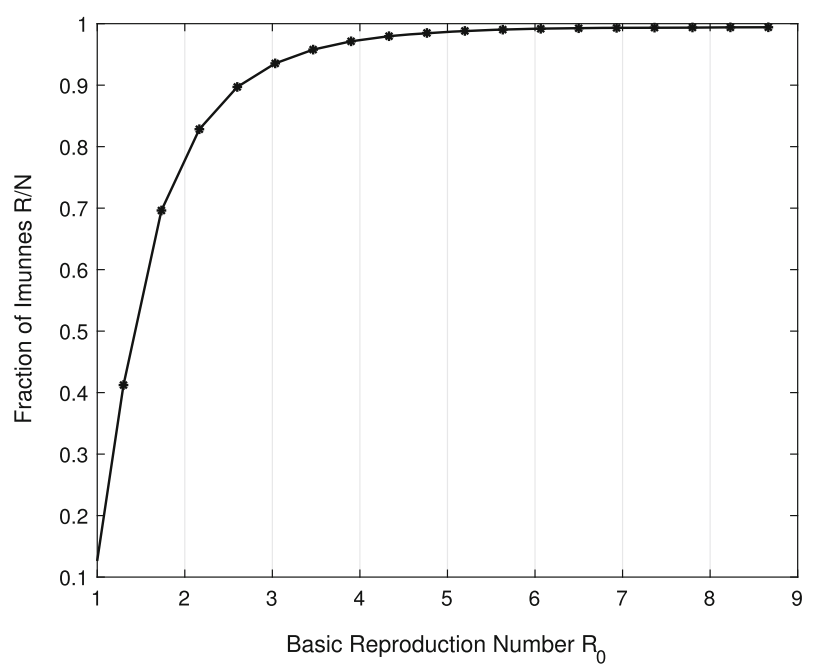

Fig. 10 The fraction $R / N$ at the end of the first wave of the natural epidemic as a function of $R_{0}$. * indicates $R_{0}$ at which the value of $R / N$ is calculated

tions expressing enhanced cells' infection, and (2) a presence of a small amount of more virulent virus accelerating cells' infection than the original virus. In both cases, the predominance of variant virus displacing the fewer cells' infectivity virus results in the transfer to subpopulation infected with more virulent SARS-CoV-2.

Figures $5 \mathrm{~b}$ shows a good fitting for the daily recorded data of deaths. From this fitting, we retrieved the daily severe COVID-19 curve shown in Fig. 6b. The observed data situate above the retrieved curve, but the pattern is similar in both curves, indicating that the difference is the infected individuals caught by mass tests. Using the accumulated severe COVID-19 curve $\Omega$ retrieved from the estimated curve $\Theta$ shown in Fig. 6a, we estimated the accumulated deaths using the clinical evolution shown in Fig. 9b. At the end of the first wave of the epidemic, the number of fatalities will stay between 131300 and 142600 .

Yang et al. (2021a) stated that the importance of a flattened epidemic by quarantine was the avoidance of the overloaded hospitals and the time gained to develop vaccines. In Yang et al. (2020b), they showed that the relaxation retook the interrupted epidemic, approaching the natural epidemic, which is characterized by the absence of any interventions (quarantine and protective measures). We use the original virus parameters given in Table 2 to evaluate the fraction of recovered individuals at the end of the first natural epidemic (when the first plateau is reached) as a function of the basic reproduction number. Figure 10 shows the fraction $R / N$ at the end of the first wave of the natural epidemic as a function of $R_{0}$. For $R_{0}=7.63$, we have $R / N=99.38 \%$ and $S / N=0.25 \%$.

Let us discuss the role of vaccines. We discuss the effects of vaccination on the first wave of the COVID-19 epidemic, and when the natural epidemic attains a steady state. We assume that individuals vaccinated with $100 \%$ efficacy are transferred to the recovered compartment.

In Fig. 8, at the end of the first wave of the epidemic, the fraction of recovered individuals attained $82.3 \%$ of the population. On the other hand, the susceptible individuals are $15.3 \%$ of the population, including $6.7 \%$ of the isolated individuals. In Table 4 , the protection factor remained $\varepsilon=0.75$ at the end of the first wave of the epidemic. This means that by vaccinating $82.3 \%$ of the population, disregarding the past infection, the COVID-19 epidemic fades out. 
However, at this level of vaccine coverage, there is potentially retaken of the epidemic when all individuals are released and the protective measures are abandoned. In this case, a smooth outbreak may occur, approaching the trend of the natural epidemic. To void this retaken of the epidemic, around $99 \%$ of the population must be vaccinated (see Fig. 10), keeping in mind that this result refers to a vaccine with $100 \%$ efficacy. Depending on how the effectiveness of an administered vaccine is lowered, the epidemic will be sustained at a lower or intermediate level.

The basic reproduction number $R_{0}$ indicates how the epidemic grows in an outbreak, and also the level of the epidemic at the steady state (in the transmission of SARS-CoV2 without mutations, the equilibrium fraction of the susceptible individuals (at endemicity) obeys $s^{*}=1 / R_{0}$, see Eq. (33) in Appendix A.2) (Yang 2021). We stress that this relationship is not valid at the end of the first wave of the epidemic. Reaching the endemicity, the basic reproduction number $R_{0}$ determines the magnitude of effort to eradicate infection-in the case of $100 \%$ efficacy vaccine, the efforts to eliminate a disease must be vaccinating a fraction equal to or greater than $1-1 / R_{0}$ of susceptible individuals (Anderson and May 1992) to yield $R_{e f}<1$. In Yang (2001), analyzing vaccination as a control mechanism, if $R_{e f}$ is reduced by the vaccine to a value lower than one, the number of cases decreased following exponentialtype decay. For $R_{0}=7.63$, we must vaccinate at least $87 \%$ of susceptible individuals to eradicate the infection (Anderson and May 1992). For the variant SARS-CoV-2 transmission, we must vaccinate at least $88 \%$.

In this paper, we did not consider the waning of immunity. However, the loss of immunity on the COVID-19 epidemic could be negligible during the 16 months of the pandemic. We considered the same transmission rates for the original and variant viruses, and for individuals transmitting in the asymptomatic, pre-symptomatic, and mild COVID-19 compartments. This assumption can be relaxed to provide a more accurate epidemiological scenario. Finally, we can incorporate the appearance of new variants in the model, such as the currently widespread SARS-CoV-2 Delta variant originated from India.

\section{Conclusion}

During the epidemic, the fast mutations in RNA virus can result in a pool of variants. On average, in this pool, mutations behaving like lower or higher virulence than the original SARS-CoV-2 can be found. We formulated a model considering the transmission of lower (original) and higher (variant) virulent SARS-CoV-2. The original SARS-CoV-2 transmission explained the COVID-19 epidemic in São Paulo State during the partial quarantine. However, the accumulated mutations yielding variant SARS-CoV-2 changed the trend of the original COVID-19 epidemic when the relaxation began. Consequently, the severe COVID-19 cases and fatalities increased hugely.

The efficacy of COVID-19 vaccines ranges between around 50\% and 90\%. Considering this range of vaccine effectiveness, the model presented here can be adapted to evaluate vaccination schemes to maintain the transmission at a lower level or even to reach eradication. This study is left for future work.

Acknowledgements We thank to three anonymous referees for providing comments and suggestions, which contributed to improving this paper.

Author Contributions HMY: conceptualization, methodology, formal analysis, writing — original draft preparation, and supervision. LPLJ: software, data curation, and visualization. FFMC: validation and investigation. 
ACY: validation and investigation. The first draft of the manuscript was written by HMY and all the authors commented on previous versions of the manuscript. All the authors read and approved the final manuscript.

Funding This research received no specific grant from any funding agency, commercial or not-for-profit sectors.

Availability of data and material The data that support the findings of this study are openly available: São Paulo State at https://www.seade.gov.br/coronavirus/ and https://www.saopaulo.sp.gov/coronavirus/planosp.

\section{Declarations}

Conflict of interest The authors have no conflicts of interest/competing interests to declare for this study.

Ethics approval Not applicable.

Consent to participate Not applicable.

Consent for publication Not applicable.

Code availability We will provide under request.

\section{A Analysis of the model}

We present the initial and boundary conditions supplied to the system of Eqs. (2), (3), and (4) in the main text and the analysis to obtain the basic reproduction number $R_{0}$.

\section{A.1 Initial and boundary conditions}

The initial conditions (simulation time $t=0$ ) supplied to the system of Eqs. (2), (3), and (4) are

$$
S(0)=N_{0}, X(0)=n_{X}, \text { where } X=Q, E, A, P, M, D, R_{1}, R_{2}, R_{3}
$$

where $n_{X}$ is a non-negative number. For instance, $n_{E}=0$ means that there is not any exposed individuals at the beginning of the epidemic. At the beginning of the COVID-19 epidemic, we have only original virus, then we use

$$
\left\{\begin{array}{l}
\text { original }\left(S(0)=N_{0}, Q(0)=0, E(0)=30, A(0)=24\right. \\
\quad P(0)=6, M(0)=6, D(0)=1) \\
\text { and } \\
\text { variant }\left(E_{v}(0)=0, A_{v}(0)=0, P_{v}(0)=0, M_{v}(0)=0, D_{v}(0)=0\right)
\end{array}\right.
$$

plus $R_{j}(0)=0$ and $R_{j v}(0)=0$, for $j=1,2,3$. Simulation time $t=0$ corresponds to the calendar time when the first case was confirmed (February 26 for São Paulo State), and $N_{0}=44.6$ million. (See Yang et al. 2021a for details in the initial conditions' setup.)

We drop out the pulses in Eq. (2) and transfer them to the boundary conditions. Hence, equations for susceptible and isolated individuals are

$$
\left\{\begin{aligned}
\frac{\mathrm{d}}{\mathrm{d} t} S= & \phi N-\mu S-\left(\lambda+\lambda_{v}\right) S+\pi_{1} y_{1} R_{1}+\pi_{1 v} y_{1 v} R_{1 v} \\
& +\pi_{2} y_{2} R_{2}+\pi_{2 v} y_{2 v} R_{2 v}+\pi_{3} R_{3}+\pi_{3 v} R_{3 v} \\
\frac{\mathrm{d}}{\mathrm{d} t} Q= & -\mu Q
\end{aligned}\right.
$$


and the equations for original and variant SARS-CoV-2 infected individuals are given by Eqs. (3) and (4). The isolation implemented at $\tau=27$ (corresponding to calendar time March 24, $2020)$ is described by the boundary conditions

$$
\left\{\begin{array}{l}
S\left(\tau^{+}\right)=S\left(\tau^{-}\right)(1-u) \text { and } Q\left(\tau^{+}\right)=Q\left(\tau^{-}\right)+S\left(\tau^{-}\right) u \\
X\left(\tau^{+}\right)=X\left(\tau^{-}\right), \quad \text { where } X=E, A, P, M, D, R_{1}, R_{2}, R_{3}
\end{array}\right.
$$

with $\tau^{-}=\lim _{t \rightarrow \tau} t$ (for $t<\tau$ ), and $\tau^{+}=\lim _{\tau \leftarrow t} t$ (for $t>\tau$ ).

The boundary conditions supplied to the model at the moments of release $t_{i}=\tau+$ $\sum_{w=1}^{i} \tau_{w}$, for $i=1,2, \cdots$, are

$$
S\left(t_{i}^{+}\right)=S\left(t_{i}^{-}\right)+u_{i} Q_{j}\left(t_{i}^{-}\right) \text {and } Q\left(t_{i}^{+}\right)=\left(1-u_{i}\right) Q\left(t_{i}^{-}\right)
$$

plus

$$
X\left(t_{i}^{+}\right)=X\left(t_{i}^{-}\right), \text {where } X=E, A, P, M, D, R_{1}, R_{2}, R_{3}
$$

where we have $t_{i}^{-}=\lim _{t \rightarrow t_{i}} t$ (for $t<t_{i}$ ), and $t^{+}=\lim _{t_{i} \leftarrow t} t$ (for $t>t_{i}$ ). If $\tau_{w}=\tau$, then $t_{i}=\tau+i \tau$.

The boundary conditions (20), (21), and (22) are connected to the original virus's dynamic. To the variant virus's dynamic, we allow the transfer from the original virus's compartments to the variant virus's compartments ( $E, A, P$, and $M$ to corresponding $E_{v}, A_{v}, P_{v}$, and $M_{v}$ ).

\section{A.2 Trivial equilibrium point: the basic reproduction number $\boldsymbol{R}_{0}$}

The basic reproduction number $R_{0}$ is obtained by the analysis of the trivial equilibrium point in the steady state. However, the non-autonomous and varying population system of Eqs. (19), (3) and (4) does not have steady state. Nevertheless, the system of equations is autonomous if we do not consider quarantine and relaxation (we let $u=u_{i}=0, i=1,2, \cdots$ ), but the population $N$ varies. For this reason, considering the fractions of individuals in each compartment defined by

$$
x=\frac{X}{N} \text {, where } X=S, Q, E, A, P, M, D, R_{1}, R_{2}, R_{3},
$$

with similar definitions to the variant virus, and using Eq. (5) for $N$, we obtain

$$
\frac{\mathrm{d}}{\mathrm{d} t} x \equiv \frac{\mathrm{d}}{\mathrm{d} t} \frac{X}{N}=\frac{1}{N} \frac{\mathrm{d}}{\mathrm{d} t} X-x \frac{1}{N} \frac{\mathrm{d}}{\mathrm{d} t} N=\frac{1}{N} \frac{\mathrm{d}}{\mathrm{d} t} X-x(\phi-\mu)+x\left(\alpha d+\alpha_{v} d_{v}\right)
$$

Hence, the system of Eqs. (19), (3) and (4 ) in terms of fractions becomes, for susceptible and isolated individuals,

$$
\left\{\begin{aligned}
\frac{\mathrm{d}}{\mathrm{d} t} s= & \phi-\phi s-\left(\lambda+\lambda_{v}\right) s+\pi_{1} y_{1} r_{1}+\pi_{1 v} y_{1 v} r_{1 v}+\pi_{2} y_{2} r_{2}+\pi_{2 v} y_{2 v} r_{2 v} \\
& +\pi_{3} r_{3}+\pi_{3 v} r_{3 v}+s\left(\alpha d+\alpha_{v} d_{v}\right) \\
\frac{\mathrm{d}}{\mathrm{d} t} q= & -\phi q+q\left(\alpha d+\alpha_{v} d_{v}\right)
\end{aligned}\right.
$$


for original virus infected individuals,

$$
\left\{\begin{aligned}
& \frac{\mathrm{d}}{\mathrm{d} t} e=\lambda s-(\sigma+\phi) e+e\left(\alpha d+\alpha_{v} d_{v}\right) \\
& \frac{\mathrm{d}}{\mathrm{d} t} a=\left(1-j_{E}\right) l \sigma e-(\gamma+\phi) a+a\left(\alpha d+\alpha_{v} d_{v}\right) \\
& \frac{\mathrm{d}}{\mathrm{d} t} p=\left(1-j_{E}\right)(1-l) \sigma e-\left(\gamma_{1}+\phi\right) p+p\left(\alpha d+\alpha_{v} d_{v}\right) \\
& \frac{\mathrm{d}}{\mathrm{d} t} m=\left(1-j_{A}\right)(1-\chi) \gamma a+\left(1-j_{P}\right) k \gamma_{1} p-\left(\gamma_{3}+\phi\right) m+m\left(\alpha d+\alpha_{v} d_{v}\right) \\
& \frac{\mathrm{d}}{\mathrm{d} t} d=\left(1-j_{P}\right)(1-k) \gamma_{1} p-\left(\gamma_{2}+\phi+\alpha\right) d+d\left(\alpha d+\alpha_{v} d_{v}\right) \\
& \frac{\mathrm{d}}{\mathrm{d} t} r_{1}=\left(1-j_{A}\right) \chi \gamma a+\left(1-j_{M}\right) \gamma_{3} m+\gamma_{2} d-\left(\pi_{1}+\phi\right) r_{1}+r_{1}\left(\alpha d+\alpha_{v} d_{v}\right) \frac{\mathrm{d}}{\mathrm{d} t} r_{2}=\left(1-y_{1}\right) \pi_{1} r_{1}-\left(\pi_{2}+\phi\right) r_{2}+r_{2}\left(\alpha d+\alpha_{v} d_{v}\right) \\
& \frac{\mathrm{d}}{\mathrm{d} t} r_{3}=\left(1-y_{2}\right) \pi_{2} r_{2}-\left(\pi_{3}+\phi\right) r_{3}+r_{3}\left(\alpha d+\alpha_{v} d_{v}\right),
\end{aligned}\right.
$$

and for variant virus infected individuals,

$$
\left\{\begin{aligned}
\frac{\mathrm{d}}{\mathrm{d} t} e_{v}= & \lambda_{v} s-\left(\sigma_{v}+\phi\right) e_{v}+e_{v}\left(\alpha d+\alpha_{v} d_{v}\right) \\
\frac{\mathrm{d}}{\mathrm{d} t} a_{v}= & j_{E} l \sigma e+l_{v} \sigma_{v} e_{v}-\left(\gamma_{v}+\phi\right) a_{v}+a_{v}\left(\alpha d+\alpha_{v} d_{v}\right) \\
\frac{\mathrm{d}}{\mathrm{d} t} p_{v}= & j_{E}(1-l) \sigma e+\left(1-l_{v}\right) \sigma_{v} e_{v}-\left(\gamma_{1 v}+\phi\right) p_{v}+p_{v}\left(\alpha d+\alpha_{v} d_{v}\right) \\
\frac{\mathrm{d}}{\mathrm{d} t} m_{v}= & j_{A}(1-\chi) \gamma a+j_{P} k \gamma_{1} p+\left(1-\chi_{v}\right) \gamma_{v} a_{v}+k_{v} \gamma_{1 v} p_{v}-\left(\gamma_{3 v}+\phi\right) m_{v} \\
& +m_{v}\left(\alpha d+\alpha_{v} d_{v}\right) \\
\frac{\mathrm{d}}{\mathrm{d} t} d_{v}= & j_{P}(1-k) \gamma_{1} p+\left(1-k_{v}\right) \gamma_{1 v} p_{v}-\left(\gamma_{2 v}+\phi+\alpha_{v}\right) d_{v}+d_{v}\left(\alpha d+\alpha_{v} d_{v}\right) \\
\frac{\mathrm{d}}{\mathrm{d} t} r_{1 v}= & j_{A} \chi \gamma a+j_{M} \gamma_{3} m+\chi_{v} \gamma_{v} a_{v}+\gamma_{3 v} m_{v}+\gamma_{2 v} d_{v}-\left(\pi_{1 v}+\phi\right) r_{1 v} \\
& +r_{1 v}\left(\alpha d+\alpha_{v} d_{v}\right) \\
\frac{\mathrm{d}}{\mathrm{d} t} r_{2 v}= & \left(1-y_{1 v}\right) \pi_{1 v} r_{1 v}-\left(\pi_{2 v}+\phi\right) r_{2 v}+r_{2 v}\left(\alpha d+\alpha_{v} d_{v}\right) \\
\frac{\mathrm{d}}{\mathrm{d} t} r_{3 v}= & \left(1-y_{2 v}\right) \pi_{2 v} r_{2 v}-\left(\pi_{3 v}+\phi\right) r_{3 v}+r_{3 v}\left(\alpha d+\alpha_{v} d_{v}\right)
\end{aligned}\right.
$$

where $\lambda$ is the force of infection given by Eq. (1) in the main text re-written as

$$
\lambda=\varepsilon\left(\beta_{1} a+\beta_{2} p+\beta_{3} z m\right) \text {, and } \lambda_{v}=\varepsilon\left(\beta_{1 v} a_{v}+\beta_{2 v} p_{v}+\beta_{3 v} z_{v} m_{v}\right),
$$

and

$s+q+e+a+p+m+d+r_{1}+r_{2}+r_{3}+e_{v}+a_{v}+p_{v}+m_{v}+d_{v}+r_{1 v}+r_{2 v}+r_{3 v}=1$.

This new system of equations has a steady state, that is, the number of individuals in all classes varies with time; however, their fractions attain equilibrium values (the sum of derivatives of all classes is zero). 
The trivial (disease-free) equilibrium point $P^{0}$ of the new system of Eqs. (23), (24) and (25) is given by

$$
P^{0}=\left(s^{0}=1, \mathbf{x}^{0}=0\right),
$$

where $\mathbf{x}^{0}=\left(q, e, a, p, m, d, r_{1}, r_{2}, r_{3}, e_{v}, a_{v}, p_{v}, m_{v}, d_{v}, r_{1 v}, r_{2 v}, r_{3 v}\right)$. Let us assess the stability of $P^{0}$ by applying the next generation matrix theory considering the vector of variables $x=\left(e, a, p, m, e_{v}, a_{v}, p_{v}, m_{v}\right)$ (Diekmann et al.2010). We apply method proposed in Yang (2014) and proved in Yang and Greenhalgh (2015). There are control mechanisms, hence we obtain the reduced reproduction number $R_{c}$ by interventions.

To obtain the reduced reproduction number, diagonal matrix $V$ is considered. Hence, the vectors $f$ and $v$ are

$$
f^{T}=\left(\begin{array}{c}
\varepsilon\left(\beta_{1} a+\beta_{2} p+\beta_{3} z m\right) s+e\left(\alpha d+\alpha_{v} d_{v}\right) \\
\left(1-j_{E}\right) l \sigma e+a\left(\alpha d+\alpha_{v} d_{v}\right) \\
\left(1-j_{E}\right)(1-l) \sigma e+p\left(\alpha d+\alpha_{v} d_{v}\right) \\
\left(1-j_{A}\right)(1-\chi) \gamma a+\left(1-j_{P}\right) k \gamma_{1} p+m\left(\alpha d+\alpha_{v} d_{v}\right) \\
\varepsilon\left(\beta_{1 v} a_{v}+\beta_{2 v} p_{v}+\beta_{3 v} z_{v} m_{v}\right) s+e_{v}\left(\alpha d+\alpha_{v} d_{v}\right) \\
j_{E} l \sigma e+l_{v} \sigma_{v} e_{v}+a_{v}\left(\alpha d+\alpha_{v} d_{v}\right) \\
j_{E}(1-l) \sigma e+\left(1-l_{v}\right) \sigma_{v} e_{v}+p_{v}\left(\alpha d+\alpha_{v} d_{v}\right) \\
j_{A}(1-\chi) \gamma a+j_{P} k \gamma_{1} p+\left(1-\chi_{v}\right) \gamma_{v} a_{v}+k_{v} \gamma_{1 v} p_{v}+m_{v}\left(\alpha d+\alpha_{v} d_{v}\right)
\end{array}\right)
$$

and

$$
v^{T}=\left(\begin{array}{c}
(\sigma+\phi) e \\
(\gamma+\phi) a \\
\left(\gamma_{1}+\phi\right) p \\
\left(\gamma_{3}+\phi\right) m \\
\left(\sigma_{v}+\phi\right) e_{v} \\
\left(\gamma_{v}+\phi\right) a_{v} \\
\left(\gamma_{1 v}+\phi\right) p_{v} \\
\left(\gamma_{3 v}+\phi\right) m_{v}
\end{array}\right)
$$

where the superscript $T$ stands for the transposition of a matrix, from which we obtain the matrices $F$ and $V$ (see Diekmann et al. 2010) evaluated at the trivial equilibrium $P^{0}$, which were omitted. The next-generation matrix $F V^{-1}$ is 


$$
F V^{-1}=\left[\begin{array}{cccccccc}
0 & \frac{\varepsilon \beta_{1}}{\gamma+\phi} & \frac{\varepsilon \beta_{2}}{\gamma_{1}+\phi} & \frac{\varepsilon z \beta_{3}}{\gamma_{3}+\phi} & 0 & 0 & 0 & 0 \\
\frac{\left(1-j_{E}\right) l \sigma}{\sigma+\phi} & 0 & 0 & 0 & 0 & 0 & 0 & 0 \\
\frac{\left(1-j_{E}\right)(1-l) \sigma}{\sigma+\phi} & 0 & 0 & 0 & 0 & 0 & 0 & 0 \\
0 & \frac{\left(1-j_{A}\right)(1-\chi) \gamma}{\gamma+\phi} & \frac{\left(1-j_{P}\right) k \gamma_{1}}{\gamma_{1}+\phi} & 0 & 0 & 0 & 0 & 0 \\
0 & 0 & 0 & 0 & 0 & \frac{\varepsilon \beta_{1 v}}{\gamma_{v}+\phi} & \frac{\varepsilon \beta_{2 v}}{\gamma_{1 v}+\phi} & \frac{\varepsilon z_{v} \beta_{3 v}}{\gamma_{3 v}+\phi} \\
\frac{j_{E} l \sigma}{\sigma+\phi} & 0 & 0 & 0 & \frac{l_{v} \sigma_{v}}{\sigma_{v}+\phi} & 0 & 0 & 0 \\
\frac{j_{E}(1-l) \sigma}{\sigma+\phi} & 0 & 0 & 0 & \frac{\left(1-l_{v}\right) \sigma_{v}}{\sigma_{v}+\phi} & 0 & 0 & 0 \\
0 & 0 & 0 & 0 & 0 & \frac{\left(1-\chi_{v}\right) \gamma_{v}}{\gamma_{v}+\phi} & \frac{k_{v} \gamma_{1 v}}{\gamma_{1 v}+\phi} & 0
\end{array}\right]
$$

and the characteristic equation corresponding to $F V^{-1}$ is

$$
\left[\varkappa^{4}-\left(R_{1}+R_{2}\right) \varkappa^{2}-R_{3} \varkappa\right] \times\left[\varkappa^{4}-\left(R_{1 v}+R_{2 v}\right) \varkappa^{2}-R_{3 v} \varkappa\right]=0,
$$

where the partial reproduction numbers $R_{1}, R_{2}, R_{3}, R_{1 v}, R_{2 v}$ and $R_{3 v}$ are

$$
\left\{\begin{array}{l}
R_{1}=\frac{\left(1-j_{E}\right) l \sigma}{\sigma+\phi} \frac{\varepsilon \beta_{1}}{\gamma+\phi} \\
R_{2}=\frac{\left(1-j_{E}\right)(1-l) \sigma}{\sigma+\phi} \frac{\varepsilon \beta_{2}}{\gamma_{1}+\phi} \\
R_{3}=\left[\frac{\left(1-j_{E}\right) l \sigma}{\sigma+\phi} \frac{\left(1-j_{A}\right)(1-\chi) \gamma}{\gamma+\phi}+\frac{\left(1-j_{E}\right)(1-l) \sigma\left(1-j_{P}\right) k \gamma_{1}}{\sigma+\phi}\right] \frac{\varepsilon z \beta_{3}}{\gamma_{1}+\phi} \\
R_{1 v}=\frac{l_{v} \sigma_{v}}{\sigma_{v}+\phi} \frac{\varepsilon \beta_{1 v}}{\gamma_{v}+\phi} \\
R_{2 v}=\frac{\left(1-l_{v}\right) \sigma_{v}}{\sigma_{v}+\phi} \frac{\varepsilon \beta_{2 v}}{\gamma_{1 v}+\phi} \\
R_{3 v}=\left[\frac{l_{v} \sigma_{v}}{\sigma_{v}+\phi} \frac{\left(1-\chi_{v}\right) \gamma_{v}}{\gamma_{v}+\phi}+\frac{\left(1-l_{v}\right) \sigma_{v}}{\sigma_{v}+\phi} \frac{k_{v} \gamma_{1 v}}{\gamma_{1 v}+\phi}\right] \frac{\varepsilon z_{v} \beta_{3 v}}{\gamma_{3 v}+\phi} .
\end{array}\right.
$$

We have two third-degree polynomials, and the evaluation of the spectral radius $\rho\left(F V^{-1}\right)$ is not an easy task. The procedure proposed in Yang (2014) allows us to obtain the threshold $R_{c}$ using the sum of coefficients of the characteristic equations, that is,

$$
R_{c}=\max \left\{R, R_{v}\right\},
$$

where $R$ and $R_{v}$ are given by

$$
R=R_{1}+R_{2}+R_{3} \text {, and } R_{v}=R_{1 v}+R_{2 v}+R_{3 v} .
$$

Hence, the trivial equilibrium point $P^{0}$ is locally asymptotically stable if $R_{c}<1$.

The control parameter $\varepsilon$ decreases the transmission rate, which is absent at the beginning of the epidemic, and the definition of the basic reproduction number is fulfilled. Hence, the stringent definition of the basic reproduction number $R_{0}$ is obtained when we let $\varepsilon=1$ (absence of protective measures) in Eq. (30), and $R_{c}=R_{0}$. Moreover, at the beginning of the COVID-19 epidemic, the variant virus is absent $\left(R_{v}=0\right)$, hence $R=R_{0}$.

In this model, it is not an easy task to obtain the fraction of the susceptible individual at the non-trivial equilibrium point by solving the system of Eqs. (23), (24) and (25), neither by calculating the characteristic equation applying the procedure proposed in Yang (2014). 
Hence, we can not obtain the effective reproduction number $R_{\text {ef }}$ (see Yang et al. 2021a). However, for original SARS-CoV-2 transmission alone, letting $R_{v}=0$, we have

$$
s^{*} \approx \frac{S^{*}}{N^{*}}=\frac{1}{R_{0}} \text { and } R_{e f} \approx R_{0} \frac{S}{N} .
$$

The global stability of the trivial equilibrium point when $R_{0}<1$ can be found in Yang et al. (2021b).

The basic reproduction number $R_{0}$ is the secondary cases produced by one infectious individual (could be anyone in one of the individuals harboring the virus) in a completely susceptible population without constraints (Anderson and May 1992). Let us understand $R_{1}$, $R_{2}$, and $R_{3}$ stressing that the interpretation is the same for the mutant variant. To facilitate the understanding, we consider this infectious individual in exposed class $E$. This individual enters into one of the infectious compartments composed of asymptomatic $(A)$, pre-symptomatic $(P)$; and pre-symptomatic individual enters into mild COVID-19 compartment $(M)$.

1. $R_{1}$ (and $R_{2}$ ) takes into account the transmission by one exposed individual becoming asymptomatic $(A)$ or pre-symptomatic $(P)$. We interpret for asymptomatic individual transmitting (between parentheses, for pre-symptomatic individual) infection. One exposed individual survives during the incubation period with probability $\sigma /(\sigma+\phi)$ and does not harbor virulent variant with probability $1-j_{E}$, and becomes asymptomatic with probability $l$ (pre-symptomatic, with $1-l$ ) and generates, during the time $1 /(\gamma+\phi)$ (pre-symptomatic, $\left.1 /\left(\gamma_{1}+\phi\right)\right)$ staying in this class, on average $\beta_{1} /(\gamma+\phi)$ (pre-symptomatic, $\beta_{2} /(\gamma+\phi)$ ) secondary cases, which is $R_{1}$ (and $R_{2}$ ).

2. $R_{3}$ takes into account the transmission by a mild COVID-19 individual. An infectious individual has two routes to reach $M$ : passing through $A$ or $P$ (this case is given between parentheses). One exposed individual survives during the incubation period with probability $\sigma /(\sigma+\phi)$, and not harboring virulent variant with probability $1-j_{E}$, and becomes asymptomatic with probability $l_{v}$ and not harboring virulent variant with probability $1-j_{A}$ (pre-symptomatic, with $1-l_{v}$ and $1-j_{P}$ ); survives at this stage with probability $\gamma /(\gamma+\phi)$ (pre-symptomatic, $\left.\gamma_{1} /\left(\gamma_{1}+\phi\right)\right)$ and presents mild COVID-19 $(M)$ with probability $1-\chi$ (pre-symptomatic, $k$ ); and generates, during the time $1 /\left(\gamma_{3}+\phi\right)$ staying in this class, on average $z \beta_{3} /\left(\gamma_{3}+\phi\right)$ secondary cases, which is $R_{3}$.

\section{B Estimation method}

The detailed estimation procedure can be found in Yang et al. (2021a). Briefly, to estimate the model parameters, we consider the squared sum

$$
\sum_{i=1}^{n}\left[\digamma\left(t_{i}\right)-\digamma^{o b}\left(t_{i}\right)\right]^{2},
$$

where $\digamma\left(t_{i}\right)$ is the solution of the dynamic system and $\digamma^{o b}\left(t_{i}\right)$ is the observed value at time $t_{i}$ :

(1) To estimate $\beta_{1}, \beta_{2}, \beta_{3}, u$, and $\varepsilon$, we use $\digamma\left(t_{i}\right)=\Omega$ given by Eq. (7) and $\digamma^{o b}=\Omega^{o b}$.

(2) To estimate $\alpha$, we use $\digamma\left(t_{i}\right)=\Theta\left(t_{i}+\Delta\right)$ given by Eq. (9) and $\digamma^{o b}=\Theta^{o b}$. We considered $\Delta=15$ days obtained by analyzing the data from São Paulo State (Yang et al. 2021a). 
(3) To estimate the fatality proportions and the progression rates of COVID-19, we use $\digamma\left(t_{i}\right)=\Theta\left(t_{i}+\Delta_{1}\right)$ given by Eqs. (14), (15), and (16) and $\digamma^{o b}=\Theta^{o b}$. We must consider that the time of registering new cases and deaths is delayed by $\Delta_{1}$ days, that is, $\Theta_{1 o}\left(t+\Delta_{1}\right)=\alpha_{1 o} \varsigma_{1 o} B_{1 o}(t)$, for instance.

All model parameters are described in Yang et al. (2021a), except the hospitalization parameters' values. Let us present their values, considering three clinical stages in the COVID-19 states: (I) early infection, (II) pulmonary phase, and (III) hyper inflammation phase. Siddiqi and Mehra (2020) observed that complications appeared around 10-12 days after initial symptoms, leading to the cytokine storm. Hence, we let for the period in each one of the three phases $\varsigma_{1 o}^{-1}=3, \varsigma_{2 o}^{-1}=7$ and $\varsigma_{3 o}^{-1}=15$ (all in days). We allow increased periods for variant subpopulation, $\varsigma_{1 o}^{-1}=4, \varsigma_{2 o}^{-1}=9$ and $\varsigma_{3 o}^{-1}=20$ (all in days).

At the beginning of the epidemic, severe COVID-19 cases $(D)$ were confirmed during hospital care; hence, we use $h_{o}=1.0$ and $h_{v}=1.0$. In Wuhan, China (Surveillances 2020), $81 \%$ of infections did not need hospital care, $14 \%$ were severe (developing severe diseases including pneumonia and shortness of breath), and $4.7 \%$ were critical (respiratory failure, septic shock, and multi-organ failure). From $19 \%$, we use $h_{1}=0.19$, which is the proportion of hospitalized individuals. However, we use higher values for variant and original subpopulations, $h_{1 v}=0.25$ and $h_{1 o}=0.21$. For the ratio hospital:ICUs/intubated, we use approximately $14 \%$ and $4.7 \%$, resulting in $3: 1$, and $h_{2}=1 / 4=0.25$, which is the proportion of ICUs/intubated care. However, we use higher values for variant and original subpopulations, $h_{2 v}=0.5$ and $h_{2 o}=0.45$. We assume a low mortality rate in viremia (phase 1), letting $\alpha_{1 o}=0.0005$ and $\alpha_{1 v}=0.0005$. For patients with an inflammatory response in-hospital care (phase 2), we let $\alpha_{2 o}=0.04$ and $\alpha_{2 v}=0.04$. Finally, we assume that proportions not surviving in ICUs/intubated with Cytokine storm (phase 3) are $\alpha_{3 o}=0.55$ and $\alpha_{3 v}=0.55$.

\section{References}

Alvarenga MY, Sameshima K, Baccalá LA, Yang HM (1999) Non-linear analysis of the rhythmic activity in rodent brains. Math Biosci 157(1-2):287-302

Anderson RM, May RM (1992) Infectious diseases of humans: dynamics and control. Oxford University Press, Oxford

Battineni G, Chintalapudi N, Amenta F (2020) SARS-CoV-2 epidemic calculation in Italy by SEIR compartmental models. Appl Comput Inform 16/17: 1-10. https://doi.org/10.1108/ACI-09-2020-0060

Boletim completo (2020). https://www.seade.gov.br/coronavirus/. Accessed 8 May 2020

CDC: Sars-cov-2 variants (2021). https://www.cdc.gov/coronavirus/2019-ncov/cases-updates/variantsurveillance/variant-info.html. Accessed 23 February

Diekmann O, Heesterbeek J, Roberts MG (2010) The construction of next-generation matrices for compartmental epidemic models. J R Soc Interface 7(47):873-885

Ferguson NM, Laydon D, Nedjati-Gilani G, Imai N, Ainslie K, Baguelin M, Bhatia S, Boonyasiri A, Cucunubá Z, Cuomo-Dannenburg G et al (2020) Impact of non-pharmaceutical interventions (NPIS) to reduce covid-19 mortality and healthcare demand. Imperial College covid-19 response team. Imperial College COVID-19 Response Team, p 20

Guirao A (2020) The covid-19 outbreak in Spain. A simple dynamics model, some lessons, and a theoretical framework for control response. Infect Dis Model 5:652-669

Informações covid-19 (2021). http://www.transparencia.am.gov.br/covid-19/monitoramento-covid-19/. Accessed 27 February

Korber B, Fischer WM, Gnanakaran S, Yoon H, Theiler J, Abfalterer W, Hengartner N, Giorgi EE, Bhattacharya $\mathrm{T}$, Foley B et al (2020) Tracking changes in sars-cov-2 spike: evidence that d614g increases infectivity of the covid-19 virus. Cell 182(4):812-827

Liu Y, Ning Z, Chen Y, Guo M, Liu Y, Gali NK, Sun L, Duan Y, Cai J, Westerdahl D et al (2020) Aerodynamic analysis of Sars-cov-2 in two Wuhan hospitals. Nature 582(7813):557-560 
Pericàs J, Hernandez-Meneses M, Sheahan T, Quintana E, Ambrosioni J, Sandoval E, Falces C, Marcos M, Tuset M, Vilella A et al (2020) Covid-19: from epidemiology to treatment. Eur Heart J 41(22):2092-2112

Plano são paulo (2020). https://www.saopaulo.sp.gov.br/coronavirus/planosp. Accessed 10 May 2020

Siddiqi HK, Mehra MR (2020) Covid-19 illness in native and immunosuppressed states: a clinical-therapeutic staging proposal. J Heart Lung Transpl 39(5):405

Surveillances V (2020) The epidemiological characteristics of an outbreak of 2019 novel coronavirus diseases (covid-19)—China, 2020. China CDC Wkly 2(8):113-122

WHO (2021) Coronavirus disease (covid-19): Virus evolution, 30 December 2020. https://www.who.int/newsroom/q-a-detail/sars-cov-2-evolution. Accessed 23 Feb 2020

Yang $H$ (2001) Modeling directly transmitted infections in a routinely vaccinated population-the force of infection described by a Volterra integral equation. Appl Math Comput 122(1):27-58

Yang H (2014) The basic reproduction number obtained from Jacobian and next generation matrices-a case study of dengue transmission modelling. Biosystems 126:52-75

Yang HM (2021) Are the beginning and ending phases of epidemics characterized by the next generation 750 matrices? -A case study of drug-sensitive and resistant tuberculosis model. J Biol Syst 29(3):719-740

Yang H, Greenhalgh D (2015) Proof of conjecture in: the basic reproduction number obtained from Jacobian and next generation matrices-a case study of dengue transmission modelling. Appl Math Comput 265:103-107

Yang HM, Lombardi Junior HMLP, Yang AC (2020a) Are the SIR and SEIR models suitable to estimate the basic reproduction number for the covid-19 epidemic? medRxiv. https://doi.org/10.1101/2020.10. 11.20210831

Yang HM, Lombardi Junior LP, Yang AC, Castro FFM (2020b) Evaluating the impacts of release in Säo Paulo state (Brazil) on the epidemic of covid-19 based on mathematical model. medRxiv. https://doi.org/10. $1101 / 2020.08 .03 .20167221$

Yang HM, Lombardi Junior LP, Castro FFM, Yang AC (2021a) Mathematical modeling of the transmission of Sars-cov-2-evaluating the impact of isolation in São Paulo state (Brazil) and lockdown in Spain associated with protective measures on the epidemic of covid-19. Plos One 16(6):e0252271

Yang HM, Junior LLP, Yang AC (2021b) Modeling the transmission of the new coronavirus in São Paulo state, Brazil - assessing the epidemiological impacts of isolating young and elder persons. Math Med Biol J IMA 38(2):137-177

Zhu N, Zhang D, Wang W, Li X, Yang B, Song J, Zhao X, Huang B, Shi W, Lu R et al (2020) A novel coronavirus from patients with pneumonia in china, 2019. New Engl J Med 382(8):727-733. https://doi. org/10.1056/NEJMoa2001017

Publisher's Note Springer Nature remains neutral with regard to jurisdictional claims in published maps and institutional affiliations. 